\title{
Symbolic Analysis for Boundary Problems: From Rewriting to Parametrized Gröbner Bases
}

\author{
Markus Rosenkranz, Georg Regensburger, Loredana Tec, and Bruno Buchberger
}

\begin{abstract}
We review our algebraic framework for linear boundary problems (concentrating on ordinary differential equations). Its starting point is an appropriate algebraization of the domain of functions, which we have named integro-differential algebras. The algebraic treatment of boundary problems brings up two new algebraic structures whose symbolic representation and computational realization is based on canonical forms in certain commutative and noncommutative polynomial domains. The first of these, the ring of integro-differential operators, is used for both stating and solving linear boundary problems. The other structure, called integro-differential polynomials, is the key tool for describing extensions of integrodifferential algebras. We use the canonical simplifier for integro-differential polynomials for generating an automated proof establishing a canonical simplifier for integro-differential operators. Our approach is fully implemented in the TH $\exists \mathrm{OREM} \forall$ system; some code fragments and sample computations are included.
\end{abstract}

Markus Rosenkranz

School of Mathematics, Statistics and Actuarial Science (SMSAS),

University of Kent, Canterbury CT2 7NF, United Kingdom,

e-mail: M.Rosenkranz@kent.ac.uk

Georg Regensburger

Johann Radon Institute for Computational and Applied Mathematics (RICAM),

Austrian Academy of Sciences, 4040 Linz, Austria,

INRIA Saclay - Île de France, Project DISCO, L2S, Supélec, 91192 Gif-sur-Yvette Cedex, France,

e-mail: Georg.Regensburgerericam.oeaw.ac.at

Loredana Tec

Research Institute for Symbolic Computation (RISC),

Johannes Kepler University, 4032 Castle of Hagenberg, Austria,

e-mail: ltecerisc.uni-linz.ac.at

Bruno Buchberger

Research Institute for Symbolic Computation (RISC),

Johannes Kepler University, 4032 Castle of Hagenberg, Austria,

e-mail: Bruno.Buchberger@risc.uni-linz.ac.at 


\section{Introduction}

Overall View. When problems from Analysis-notably differential equations-are treated by methods from Symbolic Computation, one speaks of Symbolic Analysis, as in the eponymous workshops of the FoCM conference series [34]. Symbolic Analysis is based on algebraic structures, as all other symbolic branches, but its special flavor comes from its connection with analytic and numeric techniques. As most differential equations arising in the applications can only be solved numerically, this connection is absolutely vital.

If symbolic techniques cannot solve "most" differential equations, what else can they do? The answers are very diverse (reductions, normal forms, symmetry groups, singularity analysis, triangularization etc), and in the frame of this paper we can only point to surveys like [79] and [36, §2.11]. In fact, even the notion of "solving" is quite subtle and can be made precise in various ways. Often a symbolic method will not provide the "solution" in itself but valuable information about it to be exploited for subsequent numerical simulation.

Our own approach takes a somewhat intermediate position while diverging radically in another respect: Unlike most other symbolic methods known to us, we consider differential equations along with their boundary conditions. This is not only crucial for many applications, it is also advantageous from an algebraic point of view: It allows to define a linear operator, called the Green's operator, that maps the so-called forcing function on the right-hand side of an equation to the unique solution determined by the boundary conditions. This gives rise to an interesting structure on Green's operators and on boundary problems (Sect. 5). Algebraically, the consequence is that we have to generalize the common structure of differential algebras to what we have called integro-differential algebras (Sect. 3).

Regarding the solvability issues, the advantage of this approach is that it uncouples the task of finding an algebraic representation of the Green's operator from that of carrying out the quadratures involved in applying the Green's operator to a forcing function. While the latter may be infeasible in a symbolic manner, the former can be done by our approach (with numerical quadratures for integrating forcing functions).

The research program just outlined has been pursued in the course of the SFB project $\mathrm{F} 013$ (see below for a brief chronology), and the results have been reported elsewhere $[70,73,66]$. For the time being, we have restricted ourselves to linear boundary problems, but the structure of integro-differential polynomials [72] may be a first stepping stone towards nonlinear Green's operators. Since the algebraic machinery for Green's operators is very young, our strategy was to concentrate first on boundary problems for ordinary differential equations (ODEs), with some first steps towards partial differential equations (PDEs) undertaken more recently [74]. For an application of our methods in the context of actuarial mathematics, we refer to [2], for a more algebraic picture from the skew-polynomial perspective see [67].

New Results. In the present paper, we will present a new confluence proof for the central data structure used in our approach: As the algebraic language for Green's 
operators, the integro-differential operators (Sect. 4) are defined as a ring of noncommutative polynomials in infinitely many variables, modulo an infinitely generated ideal. While the indeterminates represent the basic operations of analysis (differentiation, integration, extraction of boundary values and multiplication by one of infinitely many coefficient functions), this ideal specifies their interaction (e.g. the fundamental theorem of calculus describing how differentiation and integration interact). Our new proof is fully automated within the $\mathrm{TH} \exists \mathrm{OREM} \forall$ system (Sect. 2), using a generic noncommutative polynomial reduction based on a noncommutative adaption of reduction rings [23]; see also [83] for a short outline of the proof.

In a way, the new proof completes the circle started with the ad-hoc confluence proof in [69]. For the latter, no algebraic structure was available for coping with certain expressions that arise in the proof because they involved generic coefficient functions along with their integrals and derivatives (rather than the operator indeterminates modeling integration and differentiation!), while this structure is now provided by the afore-mentioned integro-differential polynomials (Sect. 6). Roughly speaking, this means within the spectrum between rewrite systems (completion by the Knuth-Bendix procedure) and Gröbner bases (completion by Buchberger's algorithm), we have moved away from the former towards the latter [20]. We will come back to this point later (Sect. 7).

Moreover, the paper includes the following improvements and innovations: The setting for Gröbner bases and the Buchberger algorithm are introduced generically for commutative and noncommutative rings (allowing infinitely many variables and generators), based on reduction rings and implemented in the TH $\exists$ OREM $\forall$ system (Sect. 2). The presentation of integro-differential algebras is streamlined and generalized (Sect. 3). For both of the main computational domains-integro-differential operators and integro-differential polynomials-we have a basis free description while a choice of basis is only need for deciding equality (Sects. 4, 6). The construction of integro-differential polynomials, which was sketched in [72], is carried out in detail (Sect. 6). In particular, a complete proof of the crucial result on canonical forms (Thm. 42) is now given.

Chronological Outline. As indicated above, this paper may be seen as a kind of target line for the research that we have carried out within Project F1322 of the SFB F013 supported by the Austrian Science Fund (FWF). We have already pointed out the crucial role of analysis/numerics in providing the right inspirations for the workings of Symbolic Analysis. The development of this project is an illuminating and pleasant case in point. It was initiated by the stimulating series of Hilbert Seminars conducted jointly by Bruno Buchberger and Heinz W. Engl from October 2001 to July 2002, leading to the genesis of Project F1322 as a spin-off from Projects F1302 (Buchberger) and F1308 (Engl). Triggered by the paper [42], the idea of symbolic operator algebras emerged as a common leading theme. It engendered a vision of transplanting certain ideas like the Moore-Penrose inverse on Hilbert spaces from their homeground in functional analysis into a new domain within Symbolic Analysis, where powerful algebraic tools like Gröbner bases are 
available $[17,18,9,21]$. This vision eventually crystallized in the algebraic machinery for computing Green's operators as described before.

In the early stage of the project, those two main tools from analysis (MoorePenrose inverse) and algebra (Gröbner bases) were welded together in a rather adhoc manner, but it did provide a new tool for solving boundary problems [71]. In the course of the dissertation [69], a finer analysis led to a substantial simplification where the Moore-Penrose inverse was superseded by a purely algebraic formulation in terms of one-sided inverses and the expensive computation of a new noncommutative Gröbner basis for each boundary problem was replaced by plain reduction modulo a fixed Gröbner basis for modeling the essential operator relations. The resulting quotient algebra (called "Green's polynomials" at that time) is the precursor of the integro-differential operators described below (Sect. 4). The final step towards the current setup was the reformulation and generalization in a differential algebra setting [73] and in an abstract linear algebra setting [66].

The advances on the theoretical side were paralleled by an early implementation of the algorithm for computing Green's operators. While the ad-hoc approach with computing Gröbner bases per-problem was carried out by the help of NCAlgebra, a dedicated Mathematica package for noncommutative algebra [42], the fixed Gröbner basis for simplifying Green's operator was implemented in the TH $\exists$ OREM $\forall$ system [26]; see Sect. 2 for a general outline of this system. As the new differential algebra setting emerged, however, it became necessary to supplant this implementation by a new one. It was again integrated in the $\mathrm{TH} \exists \mathrm{OREM} \forall$ system, but now in a much more intimate sense: Instead of using a custom-tailored interface as in [69], the new package was coded directly in the TH $\exists$ OREM $\forall$ language using the elegant structuring constructs of functors [25]. Since this language is also the object language of the provers, this accomplishes the old ideal of integrating computation and deduction.

The presentation of several parts of this paper-notably Sects. 3, 4, 5-benefited greatly from a lecture given in the academic year 2009/10 on Symbolic Integral Operators and Boundary Problems by the first two authors. The lecture was associated with the Doctoral Program "Computational Mathematics: Numerical Analysis and Symbolic Computation" (W1214), which is a follow-up program to the SFB F013. We would like to thank our students for the lively discussions and valuable comments.

Overview of the Paper. We commence by having a closer look at the TH $\exists$ OREM $\forall$ system (Sect. 2), which will also be used in all sample computations presented in subsequent sections; both the sample computations and the TH $\exists$ OREM $\forall$ program code is available in an executable Mathematica notebook from www . theorema. org. We discuss canonical simplifiers for quotient structures and Gröbner bases in reduction rings, and we give a short overview of the functors used in building up the hierarchy of the algebraic structures used in the computations. The main structure among these is that of an integro-differential algebra (Sect. 3), which is the starting point for the integro-differential operators as well as the integro-differential polynomials. Since the former are, in turn, the foundation for computing Green's op- 
erators for boundary problems, we will next summarize the construction of integrodifferential operators and their basic properties (Sect. 4), while the algorithms for solving and factoring boundary problems are explained and exemplified thereafter (Sect. 5). Driving towards the focus point of this paper, we describe then the algebra of integro-differential polynomials (Sect. 6), which will be the key tool to be employed for the confluence proof. Since this proof is reduced to a computation in $\mathrm{TH} \exists \mathrm{OREM} \forall$, we will only explain the main philosophy and show some representative fragments (Sect. 7). We wind up with some thoughts about open problems and future work (Sect. 8).

\section{Data Structures for Polynomials in Theorema}

The Theorema Functor Language. The TH $\exists$ OREM $\forall$ system [26] was designed by B. Buchberger as an integrated environment for proving, solving and computing in various domains of mathematics. Implemented on top of Mathematica, its core language is a version of higher-order predicate logic that contains a natural programming language such that algorithms can be coded and verified in a unified formal frame. In this logic-internal programming language, functors are a powerful tool for building up hierarchical domains in a modular and generic way. They were introduced and first implemented in TH $\exists$ OREM $\forall$ by B. Buchberger. The general idea-and its use for structuring those domains in which Gröbner bases can be computed-is described in $[23,25]$, where one can also find references to pertinent early papers by B. Buchberger. See also [87] for some implementation aspects of functor programming.

The notion of functor in TH $\exists O R E M \forall$ is akin to functors in ML, not to be confused with the functors of category theory. From a computational point of view, a $\mathrm{TH} \exists \mathrm{OREM} \forall$ functor is a higher-order function that produces a new domain from given domains, where each domain is considered as a bundle of operations (including relations qua boolean-valued operations - in particular also carrier predicates). Operations in the new domain are defined in terms of operations in the underlying domains.

Apart from this computational aspect, functors also have an important reasoning aspect-a functor transports properties of the input domains to properties of the output domain, typical examples being the various "preservation theorems" in mathematics: "If $R$ is a ring, then $R[x]$ is also a ring". This means the functor $R \mapsto R[x]$ preserves the property of being a ring, in other words: it goes from the "category of rings" to itself. In this context, a category is simply a collection of domains characterized by a common property (a higher-order predicate on domains).

See below for an example of a functor named LexWords. It takes a linearly ordered alphabet $\mathrm{L}$ as input domain and builds the word monoid over this alphabet: 


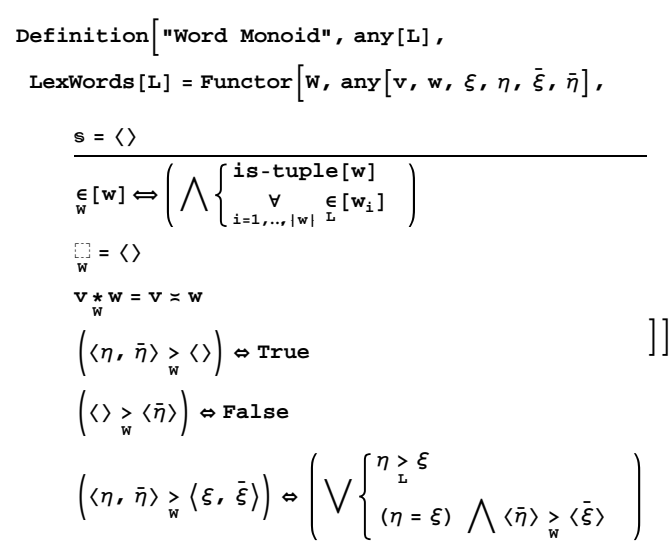

Here $\bar{\xi}, \bar{\eta}$ are sequence variables, i.e. they can be instantiated with finite sequences of terms. The new domain $W$ has the following operations: $W[\in]$ denotes the carrier predicate, the neutral element is given by $W[\square]$, the multiplication $W[*]$ is defined as concatenation, and $\mathrm{W}[>]$ defines the lexicographic ordering on $\mathrm{W}$.

In the following code fragments, we illustrate one way of building up polynomials in TH $\exists$ OREM $\forall$ starting from the base categories of fields with ordering and ordered monoids. Via the functor FreeModule, we construct first the free vector space $\mathrm{V}$ over a field $\mathrm{K}$ generated by the set of words in an ordered monoid $\mathrm{W}$. The elements of $\mathrm{V}$ are described by $\mathrm{V}[\in]$ as lists of pairs, each pair containing one (nonzero) coefficient from $\mathrm{K}$ and one basis vector from $\mathrm{W}$, where the basis vectors are ordered according to the ordering on $\mathrm{W}$. The operations of addition, subtraction and scalar multiplication are defined recursively, using the operations on $\mathrm{K}$ and $\mathrm{W}$ :

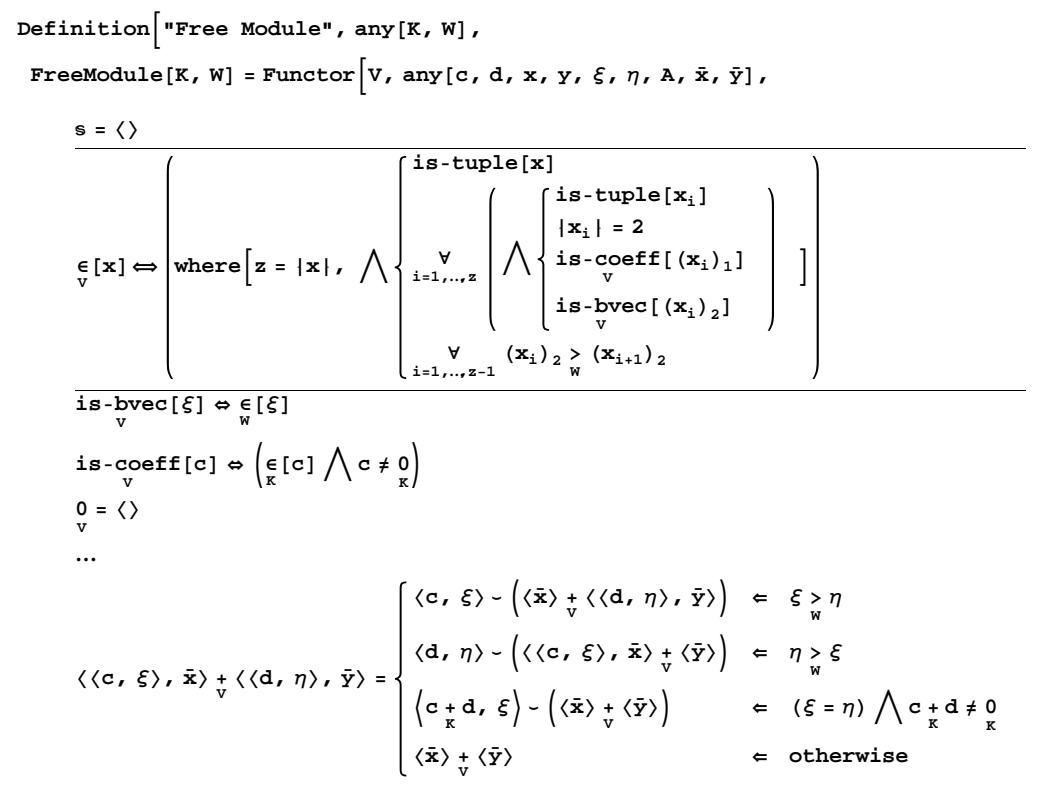




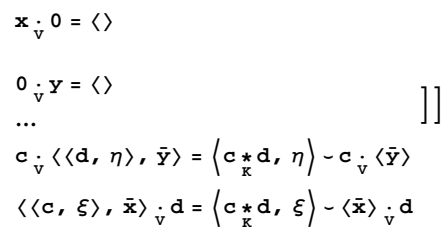

By the MonoidAlgebra functor we extend this domain, introducing a multiplication using the corresponding operations in $\mathrm{K}$ and $\mathrm{W}$ :

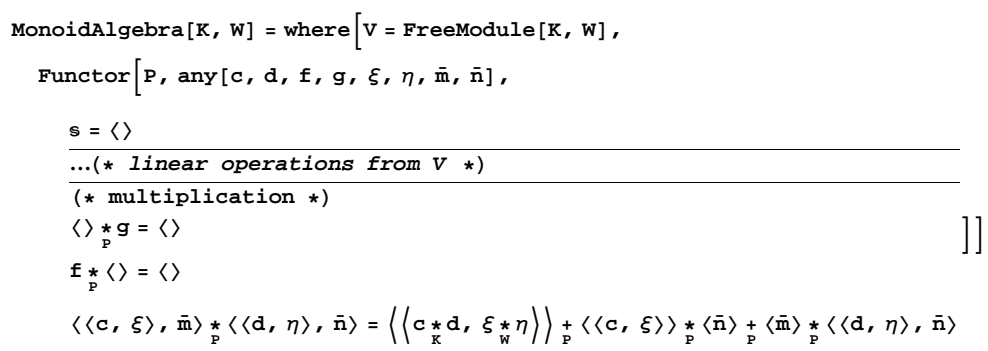

The new domain inherits the structure on the elements of V.

The main advantage of the above construction is that it is fully generic: Not only can it be instantiated for different coefficient rings (or fields) and different sets of indeterminates, it comprises also the commutative and noncommutative case (where $\mathrm{W}$ is instantiated respectively by a commutative and noncommutative monoid).

Quotient Structures and Canonical Simplifiers. In algebra (and also in the rest of mathematics), one encounters quotient structures on many occasions. The general setting is a set $A$ with various operations (an algebra in the general sense used in Sect. 6) and a congruence relation $\equiv$ on $A$, meaning an equivalence relation that is compatible with all the operations on $A$. Then one may form the quotient $A / \equiv$, which will typically inherit some properties of $A$. For example, $A / \equiv$ belongs to the category of rings if $A$ does, so we can view the quotient construction $A \mapsto A / \equiv$ as a functor on the category of rings.

But for computational purposes, the usual set-theoretic description of $A / \equiv$ as a set of equivalence classes is not suitable (since each such class is typically uncountably infinite). We will therefore use an alternative approach that was introduced in [27] as a general framework for symbolic representations. The starting point is a canonical simplifier for $A / \equiv$, meaning a map $\sigma: A \rightarrow A$ such that

$$
\sigma(a) \equiv a \quad \text { and } \quad \sigma(a)=\sigma\left(a^{\prime}\right) \text { whenever } a \equiv a^{\prime} .
$$

The set $\tilde{A}=\sigma(A)$ is called the associated system of canonical forms for $A / \equiv$.

Clearly canonical simplifiers exist for every quotient $A / \equiv$, but for computational purposes the crucial question is whether $\sigma$ is algorithmic. Depending on $A / \equiv$, it may be easy or difficult or even impossible to construct a computable $\sigma: A \rightarrow A$. In the examples that we will treat, canonical simplifiers are indeed available. 
Canonical simplifiers are also important because they allow us to compute in the quotient structure. More precisely, one can transplant the operations on $A$ to $\tilde{A}$ by defining $\omega\left(a_{1}, \ldots, a_{n}\right)=\sigma\left(\omega\left(a_{1}, \ldots, a_{n}\right)\right)$ for every operation $\omega$ on $A$. With these new operations, one may easily see that $\tilde{A}$ is isomorphic to the quotient $A / \equiv$; see the Theorem "Canonical simplification and computation" in [27, p. 13].

There is an intimate relation between canonical forms and normal forms for rewrite systems (Sect. 4 contains some basic terminology and references). In fact, every rewrite system $\rightarrow$ on an algebraic structure $A$ creates an equivalence relation $\equiv$, the symmetric closure of $\stackrel{*}{\rightarrow}$. Thus $a \equiv a^{\prime}$ if and only if $a$ and $a^{\prime}$ can be connected by an equational chain (using the rewrite rules in either direction). Typically, the relation $\equiv$ will actually be a congruence on $A$, so that the quotient $A / \equiv$ has a welldefined algebraic structure. Provided the rewrite system is noetherian, the normal forms of $\rightarrow$ are then also canonical forms for $A / \equiv$. Hence we will often identify these terms in a rewriting context.

For our implementation, we use canonical simplifiers extensively. In fact, the observation made above about computing in the quotient structure is realized by a $\mathrm{TH} \exists \mathrm{OREM} \forall$ functor, which is applied at various different places. Here $A$ is typically a $K$-algebra, with the ground field $K$ being $\mathbb{Q}$ or computable subfields of $\mathbb{R}$ and $\mathbb{C}$.

Reduction Rings and Gröbner Bases. For defining reduction on polynomials, we use the reduction ring approach in the sense of [19,23]. For commutative reduction rings, see also [81, 82]; for another noncommutative approach we refer to $[57,58$, 59].

To put it simply, a reduction ring is a ring in which Gröbner bases can be done. A full axiomatization for the commutative case is given in [19]. If such rings satisfy certain additional axioms (defining the category of so-called "Gröbner rings"), then Gröbner bases can be computed by iterated S-polynomial reduction in the given ring - this is the Gröbner Ring Extension Theorem, stated and proved in [19].

A detailed presentation of their construction in the $\mathrm{TH} \exists \mathrm{OREM} \forall$ setting was given in $[22,24]$; it is the starting point for our current work. At this point we do not give an axiomatic characterization for noncommutative reduction rings, but we do use a construction that is similar to the commutative setting. Thus we endow a polynomial domain $\mathrm{P}$, built via the MonoidAlgebra functor with word monoid $\mathrm{W}$ and field $\mathrm{K}$, with the following three operations: a noetherian (partial) ordering, a binary operation least common reducible, and a binary operation reduction multiplier. The noetherian ordering is defined in the usual way in terms of the given orderings on $\mathrm{K}$ and $\mathrm{W}$.

The basic idea of reduction multipliers is to answer the question: "With which monomial do I have to multiply a given polynomial so that it cancels the leading term of another given polynomial?" In the noncommutative case, the corresponding operation $\mathrm{rdm}$ splits into left reduction multiplier $1 \mathrm{rdm}$ and its right counterpart rrdm defined as follows:

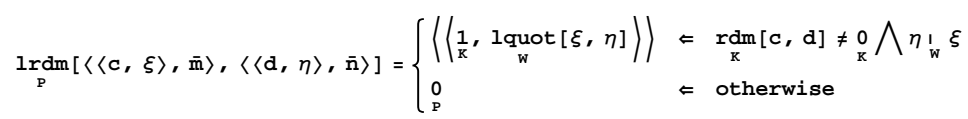




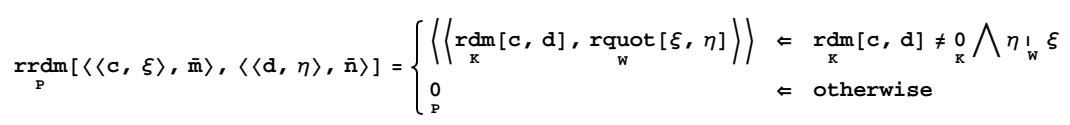

Here the divisibility relation | on $\mathrm{W}$ checks whether a given word occurs within another word, and the corresponding quotients lquot and rquot yield the word segments respectively to the left and to the right of this occurrence. Since the scalars from $\mathrm{K}$ commute with the words, it is an arbitrary decision whether one includes it in the right (as here) or left reduction multiplier. In typical cases, this scalar factor is just $r d m[c, d]=c / d$.

The operations relating Gröbner bases are introduced via a functor which is called GroebnerExtension. It defines polynomial reduction using reduction multipliers (note that this includes also the commutative case, where one actually needs only one reduction multiplier, the other one being unity):

$$
\underset{G}{\operatorname{hred}}[f, g]=f_{\bar{P}} \underset{P}{\operatorname{lrdm}}[f, g] \underset{P}{*} \underset{P}{*} \underset{P}{\operatorname{rrdm}}[f, g]
$$

The next step is to introduce reduction modulo a system of polynomials. For some applications (like the integro-differential operators described in Sect. 4), it is necessary to deal with infinite reduction systems: the polynomial ring contains infinitely many indeterminates, and reduction is applied modulo an infinite set of polynomials. In other words, we want to deal with an infinitely generated ideal in an infinitely generated algebra.

This is a broad topic, and we cannot hope to cover it in the present scope. In general one must distinguish situations where both the generators of the ideal and the algebra are parametrized by finitely many families involving finitely many parameters and more general algebras/ideals where this is not so. In the latter case, one works with finite subsets, and all computations are approximate: one never catches the whole algebraic picture. Fortunately, the applications we have in mind-in particular the integro-differential operators-are of the first type where full algorithmic control can be achieved. However, most of the common packages implementing noncommutative Gröbner bases do not support such cases [55, 56]. For some recent advances, we refer the reader to $[3,14,43,51]$ as well as Ufnarovski's extensive survey chapter [86].

Let us point out just one important class of decidable reductions in infinitely generated algebras - if an infinite set of (positively weighted) homogeneous polynomials is given, which is known to be complete for each given degree (see [51] for the proof) since one can compute a truncated Gröbner basis of such a graded ideal, which is finite up to a given degree. But if the given set is not homogeneous or cannot be clearly presented degree by degree, basically nothing can be claimed in general. Unfortunately, the applications we have in mind seem to be of this type.

In our setting, infinitely generated ideals are handled by an algorithmic operation for instantiating reduction rules. The reduction of polynomial $f$ modulo a system $\mathrm{S}$ is realized thus:

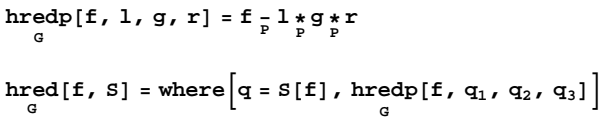


where $S[f]$ is the operation that decides if there exists g modulo which $f$ can be reduced, and it returns a triple containing the $g$ and the left/right reduction multipliers needed for performing the reduction.

The main tool for the Gröbner bases construction, namely the notion of Spolynomial, can now be defined in terms of the least common reducible:

$$
\underset{G}{\operatorname{spol}}[f, g]=\operatorname{where}[I=\underset{P}{\operatorname{lcrd}}[f, g], \underset{G}{\operatorname{hredp}}[I, f] \underset{G}{-\operatorname{hredp}}[\mathrm{L}, g]]
$$

Here $l \operatorname{crd}[f, g]$ represents the smallest monomial that can be reduced both modulo $f$ and modulo $g$, built from the least common reducible of the corresponding coefficients in $\mathrm{K}$ and the least common multiple of the words in $\mathrm{W}$ :

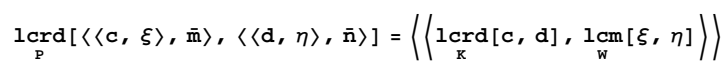

In our setting, the $1 \operatorname{crd}[\mathrm{c}, \mathrm{d}]$ can of course be chosen as unity since we work over a field $\mathrm{K}$, but in rings like $\mathbb{Z}$ one would have to use the least common multiple.

Finally, Gröbner bases are computed by the usual accumulation of S-polynomials reduction, via the following version of Buchberger algorithm [17]:

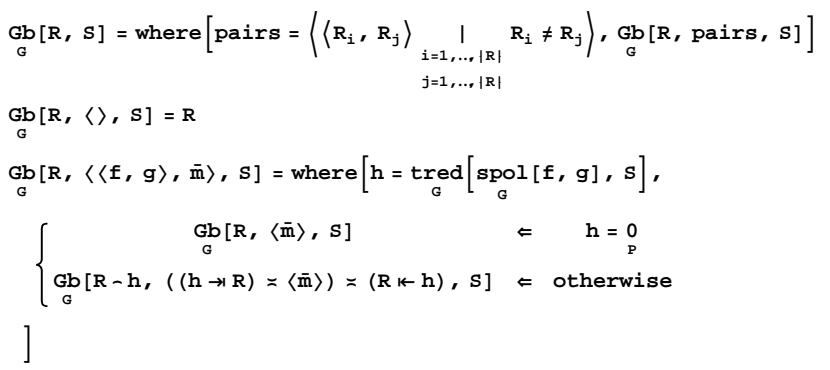

Total reduction modulo a system, denoted here by tred, is computed by iteratively performing reductions, until no more reduction is possible. The above implementation of Buchberger's algorithm is again generic since it can be used in both commutative and noncommutative settings. For finitely many indeterminates, the algorithm always terminates in the commutative case (by Dickson's Lemma); in the noncommutative setting, this cannot be guaranteed in general. For our applications we also have to be careful to ensure that the reduction systems we use are indeed noetherian (Sect. 4).

\section{Integro-Differential Algebras}

For working with boundary problems in a symbolic way, we first need an algebraic structure having differentiation along with integration. In the following definitions, one may think of our standard example $\mathscr{F}=C^{\infty}(\mathbb{R})$, where $\partial={ }^{\prime}$ is the usual derivation and $\int$ the integral operator

$$
f \mapsto \int_{a}^{x} f(\xi) d \xi
$$


for a fixed $a \in \mathbb{R}$.

Axioms and Basic Properties. Let $K$ be a commutative ring. We first recall that $(\mathscr{F}, \partial)$ is a differential $K$-algebra if $\partial: \mathscr{F} \rightarrow \mathscr{F}$ is a $K$-linear map satisfying the Leibniz rule

$$
\partial(f g)=\partial(f) g+f \partial(g) .
$$

For convenience, we may assume $K \leq \mathscr{F}$, and we write $f^{\prime}$ as a shorthand for $\partial(f)$. The following definition [73] captures the algebraic properties of the Fundamental Theorem of Calculus and Integration by Parts.

Definition 1. We call $\left(\mathscr{F}, \partial, \int\right)$ an integro-differential algebra if $(\mathscr{F}, \partial)$ is a commutative differential $K$-algebra and $\int$ is a $K$-linear section (right inverse) of $\partial$, i.e.

$$
\left(\int f\right)^{\prime}=f,
$$

such that the differential Baxter axiom

$$
\left(\int f^{\prime}\right)\left(\int g^{\prime}\right)+\int(f g)^{\prime}=\left(\int f^{\prime}\right) g+f\left(\int g^{\prime}\right)
$$

holds.

We refer to $\partial$ and $\int$ respectively as the derivation and integral of $\mathscr{F}$ and to (3) as section axiom. Moreover, we call a section $\int$ of $\partial$ an integral for $\partial$ if it satisfies (4). For the similar notion of differential Rota-Baxter algebras, we refer to [39] but see also below.

Note that we have applied operator notation for the integral; otherwise, for example, the section axiom (3) would read $\left(\int(f)\right)^{\prime}=f$, which is quite unusual at least for an analyst. We will likewise often use operator notation for the derivation, so the Leibniz rule (2) can also be written as $\partial f g=(\partial f) g+f(\partial g)$. For the future we also introduce the following convention for saving parentheses: Multiplication has precedence over integration, so $\int f \int g$ is to be parsed as $\int\left(f \int g\right)$.

Let us also remark that Definition 1 can be generalized: First, no changes are needed for the noncommutative case (meaning $\mathscr{F}$ is noncommutative). This would for example be an appropriate setting for matrices with entries in $\mathscr{F}=C^{\infty}[a, b]$, providing an algebraic framework for the results on linear systems of ODEs. Second, one may add a nonzero weight in the Leibniz axiom, thus incorporating also discrete models where $\partial$ is the difference operator defined by $(\partial f)_{k}=f_{k+1}-f_{k}$. The nice thing is that all other axioms remain unchanged. For both generalizations confer also to [39].

We study first some direct consequences of the section axiom (3). For further details on linear left and right inverses, we refer for example to [13, p. 211] or to [63] in the context of generalized inverses. We also introduce the following names for the projectors and modules associated with a section of a derivation.

Definition 2. Let $(\mathscr{F}, \partial)$ be a differential $K$-algebra and $\int$ a $K$-linear section of $\partial$. Then we call the projectors 


$$
\mathbf{J}=\int \circ \partial \quad \text { and } \quad \mathbf{E}=1-\int \circ \partial
$$

respectively the initialization and the evaluation of $\mathscr{F}$. Moreover, we refer to

$$
\mathscr{C}=\operatorname{Ker}(\partial)=\operatorname{Ker}(\mathbf{J})=\operatorname{Im}(\mathbf{E}) \quad \text { and } \quad \mathscr{I}=\operatorname{Im}\left(\int\right)=\operatorname{Im}(\mathbf{J})=\operatorname{Ker}(\mathbf{E})
$$

as the submodules of respectively constant and initialized functions.

Note that they are indeed projectors since $\mathrm{J} \circ \mathrm{J}=\int \circ\left(\partial \circ \int\right) \circ \partial=\mathrm{J}$ by (3), which implies $\mathbf{E} \circ \mathbf{E}=1-\mathbf{J}-\mathbf{J}+\mathbf{J} \circ \mathbf{J}=\mathbf{E}$. As is well known [13, p. 209], every projector is characterized by its kernel and image - they form a direct decomposition of the module into two submodules, and every such decomposition corresponds to a unique projector. We have therefore a canonical decomposition

$$
\mathscr{F}=\mathscr{C}+\mathscr{I},
$$

which allows to split off the "constant part" of every "function" in $\mathscr{F}$.

Before turning to the other axioms, let us check what all this means in the standard example $\mathscr{F}=C^{\infty}(\mathbb{R})$ with $\partial=\frac{d}{d x}$ and $\int=\int_{a}^{x}$. Obviously, the elements of $\mathscr{C}$ are then indeed the constant functions $f(x)=c$, while $\mathscr{I}$ consists of those functions that satisfy the homogeneous initial condition $f(a)=0$. This also explains the terminology for the projectors: Here $\mathbf{E} f=f(a)$ evaluates $f$ at the initialization point $a$, and $\mathrm{s} f=f-f(a)$ enforces the initial condition. Note that in this example the evaluation $\mathbf{E}$ is multiplicative; we will show below that this holds in any integrodifferential algebra.

The Leibniz rule (2) and the differential Baxter axiom (4) entail interesting properties of the two submodules $\mathscr{C}$ and $\mathscr{I}$. For understanding these, it is more economic to forget for a moment about integro-differential algebras and turn to the following general observation about projectors on an algebra. We use again operator notation, giving precedence to multiplication over the linear operators.

Lemma 3. Let $E$ and $J$ be projectors on a $K$-algebra with $E+J=1$, set

$$
C=\operatorname{Im}(E)=\operatorname{Ker}(J) \quad \text { and } \quad I=\operatorname{Ker}(E)=\operatorname{Im}(J) .
$$

Then the following statements are equivalent:

1. The projector $E$ is multiplicative, meaning $E f g=(E f)(E g)$.

2. The projector $J$ satisfies the identity $(J f)(J g)+J f g=(J f) g+f(J g)$.

3. The submodule $C$ is a subalgebra and the submodule I an ideal.

Proof. $1 . \Leftrightarrow 2$. Multiplicativity of $E=1-J$ just means

$$
f g-J f g=f g-(J f) g-f(J g)+(J f)(J g) .
$$

$\underline{1 .} \Rightarrow 3$. This follows immediately because $C$ is the image and $I$ the kernel of the algebra endomorphism $E$. 
3. $\Rightarrow 1$. Let $f, g$ be arbitrary. Since the given $K$-algebra is a direct sum of $C$ and $I$, we have $f=f_{C}+f_{I}$ and $g=g_{C}+g_{I}$ for $f_{C}=E f, g_{C}=E g \in C$ and $f_{I}=J f, g_{I}=J g \in I$. Then

$$
E f g=E f_{C} g_{C}+E f_{C} g_{I}+E f_{I} g_{C}+E f_{I} g_{I}
$$

Since $I$ is an ideal, the last three summands vanish. Furthermore, $C$ is a subalgebra, so $f_{C} g_{C} \in C$. This implies $E f_{C} g_{C}=f_{C} g_{C}$ because $E$ is a projector onto $C$.

This lemma is obviously applicable to integro-differential algebras $\mathscr{F}$ with the projectors $E=\mathbf{E}$ and $J=\mathrm{J}$ and with the submodules $C=\mathscr{C}$ and $I=\mathscr{I}$ because the differential Baxter axiom (4) is exactly condition 2 . From now on, we will therefore refer to $\mathscr{C}$ as the algebra of constant functions and to $\mathscr{I}$ as the ideal of initialized functions. Moreover, we note that in any integro-differential algebra the evaluation $\mathbf{E}=1-\int \circ \partial$ is multiplicative, meaning

$$
\mathbf{E} f g=(\mathbf{E} f)(\mathbf{E} g) \text {. }
$$

Altogether we obtain now the following characterization of integrals (note that the requirement that $\mathscr{C}$ be a subalgebra already follows from the Leibniz axiom).

Corollary 4. Let $(\mathscr{F}, \partial)$ be a differential algebra. Then a section $\int$ of $\partial$ is an integral if and only if its evaluation $\mathbf{E}=1-\int \circ \partial$ is multiplicative, and if and only if $\mathscr{I}=\operatorname{Im}\left(\int\right)$ is an ideal.

Note that the ideal $\mathscr{I}$ corresponding to an integral is in general not a differential ideal of $\mathscr{F}$. We can see this already in the standard example $C^{\infty}[0,1]$, where $\mathscr{I}$ consists of all $f \in C^{\infty}[0,1]$ with $f(0)=0$. Obviously $\mathscr{I}$ is not differentially closed since $x \in \mathscr{I}$ but $x^{\prime}=1 \notin \mathscr{I}$.

The above corollary implies immediately that an integro-differential algebra $\mathscr{F}$ can never be a field since then the only possibilities for $\mathscr{I}$ would be 0 and $\mathscr{F}$. The former case is excluded since it means that $\operatorname{Ker}(\partial)=\mathscr{F}$, contradicting the surjectivity of $\partial$. The latter case corresponds to $\operatorname{Ker}(\partial)=0$, which is not possible because $\partial 1=0$.

Corollary 5. An integro-differential algebra is never a field.

In some sense, this observation ensures that all integro-differential algebras are fairly complicated. The next result points in the same direction, excluding finitedimensional algebras.

Proposition 6. The iterated integrals $1, \int 1, \iint 1, \ldots$ are all linearly independent over K. In particular, every integro-differential algebra is infinite-dimensional.

Proof. Let $\left(u_{n}\right)$ be the sequence of iterated integrals of 1 . We prove by induction on $n$ that $u_{0}, u_{1}, \ldots, u_{n}$ are linearly independent. The base case $n=0$ is trivial. For the induction step from $n$ to $n+1$, assume $c_{0} u_{0}+\cdots+c_{n+1} u_{n+1}=0$. Applying $\partial^{n+1}$ yields $c_{n+1}=0$. But by the induction hypothesis, we have then also $c_{0}=\cdots=c_{n}=$ 0 . Hence $u_{0}, \ldots, u_{n+1}$ are linearly independent. 
Let us now return to our discussion of the differential Baxter axiom (4). We will offer an equivalent description that is closer to analysis. It is more compact but less symmetric. (In the noncommutative case one has to add the opposite versionreversing all products-for obtaining equivalence.)

Proposition 7. The differential Baxter axiom (4) is equivalent to

$$
f \int g=\int f g+\int f^{\prime} \int g
$$

in the presence of the Leibniz axiom (2) and the section axiom (3).

Proof. For proving (6) note that since $\mathscr{I}$ is an ideal, $f \int g$ is invariant under the projector $\mathrm{J}$ and thus equal to $\int\left(f \int g\right)^{\prime}=\int f^{\prime} \int g+\int f g$ by the Leibniz axiom (2) and the section axiom (3). Alternatively, one can also obtain (6) from (4) if one replaces $g$ by $\int g$ in (4). Conversely, assuming (6) we see that $\mathscr{I}$ is an ideal of $\mathscr{F}$, so Corollary 4 implies that $\int$ satisfies the differential Baxter axiom (4).

For obvious reasons, we refer to (6) as integration by parts. The usual formulation $\int f G^{\prime}=f G-\int f^{\prime} G$ is only satisfied "up to a constant", or if one restricts $G$ to $\operatorname{Im}\left(\int\right)$. Substituting $G=\int g$ then leads to (6). But note that we have now a more algebraic perspective on this well-known identity of Calculus: It tells us how $\mathscr{I}$ is realized as an ideal of $\mathscr{F}$.

Sometimes a variation of (6) is useful. Applying $\int$ to the Leibniz axiom (2) and using the fact that $\mathbf{E}=1-\mathrm{J}$ is multiplicative (5), we obtain

$$
\int f g^{\prime}=f g-\int f^{\prime} g-(\mathbf{E} f)(\mathbf{E} g),
$$

which we call the evaluation variant of integration by parts (a form that is also used in Calculus). Observe that, we regain integration by parts (6) upon replacing $g$ by $\int g$ in (7) since $\mathbf{E} \int g=0$.

Note that in general one cannot extend a given differential algebra to an integrodifferential algebra since the latter requires a surjective derivation. For example, in $\left(K\left[x^{2}\right], x \partial\right)$ the image of $\partial$ does not contain 1. As another example (cf. Sect. 6), the algebra of differential polynomials $\mathscr{F}=K\{u\}$ does not admit an integral in the sense of Definition 1 since the image of $\partial$ does not contain $u$.

How can we isolate the integro part of an integro-differential algebra? The disadvantage (and also advantage!) of the differential Baxter axiom (4) is that it entangles derivation and integral. So how can one express "integration by parts" without referring to the derivation?

Definition 8. Let $\mathscr{F}$ be a $K$-algebra and $\int$ a $K$-linear operation satisfying

$$
\left(\int f\right)\left(\int g\right)=\int f \int g+\int g \int f .
$$

Then $\left(\mathscr{F}, \int\right)$ is called a Rota-Baxter algebra (of weight zero).

Rota-Baxter algebras are named after Glen Baxter [7] and Gian-Carlo Rota [75]; see also [37, 38] for further details. In the following, we refer to (8) as Baxter axiom; 
in contrast to the differential Baxter axiom (4), we will sometimes also call it the pure Baxter axiom.

One might now think that an integro-differential algebra $\left(\mathscr{F}, \partial, \int\right)$ is a differential algebra $(\mathscr{F}, \partial)$ combined with a Rota-Baxter algebra $\left(\mathscr{F}, \int\right)$ such that the section axiom (3) is satisfied. In fact, such a structure was introduced independently by Guo and Keigher [39] under the name differential Rota-Baxter algebras. But we will see that an integro-differential algebra is a little bit more-this is why we also refer to (8) as "weak Baxter axiom" and to (4) and (6) as "strong Baxter axioms".

Proposition 9. Let $(\mathscr{F}, \partial)$ be a differential algebra and $\int$ a section for $\partial$. Then $\int$ satisfies the pure Baxter axiom (8) if and only if $\mathscr{I}=\operatorname{Im}\left(\int\right)$ is a subalgebra of $\mathscr{F}$. In particular, $\left(\mathscr{F}, \int\right)$ is a Rota-Baxter algebra for any integro-differential algebra $\left(\mathscr{F}, \partial, \int\right)$.

Proof. Clearly (8) implies that $\mathscr{I}$ is a subalgebra of $\mathscr{F}$. Conversely, if $\left(\int f\right)\left(\int g\right)$ is contained in $\mathscr{I}$, it is invariant under the projector $\mathrm{J}$ and must therefore be equal to $\int \partial\left(\int f\right)\left(\int g\right)=\int f \int g+\int g \int f$ by the Leibniz axiom (2).

So the strong Baxter axiom (4) requires that $\mathscr{I}$ be an ideal, the weak Baxter axiom (8) only that it be a subalgebra. We will soon give a counterexample for making sure that (4) is indeed asking for more than (8), see Example 14. But before this we want to express the difference between the two axioms in terms of a linearity property. Recall that both $\partial$ and $\int$ were introduced as $K$-linear operations on $\mathscr{F}$. Using the Leibniz axiom (2), one sees immediately that $\partial$ is even $\mathscr{C}$-linear. It is natural to expect the same from $\int$, but this is exactly the difference between (4) and (8).

Proposition 10. Let $(\mathscr{F}, \partial)$ be a differential algebra and $\int$ a section for $\partial$. Then $\int$ satisfies the differential Baxter axiom (4) if and only if it satisfies the pure Baxter axiom (8) and is $\mathscr{C}$-linear.

Proof. Assume first that $\int$ satisfies the differential Baxter axiom (4). Then the pure Baxter axiom (8) holds by Proposition 9. For proving $\int c g=c \int g$ for all $c \in \mathscr{C}$ and $g \in \mathscr{F}$, we use the integration-by-parts formula (6) and $c^{\prime}=0$.

Conversely, assume the pure Baxter axiom (8) is satisfied and $\int$ is $\mathscr{C}$-linear. By Proposition 7 it suffices to prove the integration-by-parts formula (6) for $f, g \in \mathscr{F}$. Since $\mathscr{F}=\mathscr{C} \dot{+} \mathscr{I}$, we may first consider the case $f \in \mathscr{C}$ and then the case $f \in \mathscr{I}$. But the first case follows from $\mathscr{C}$-linearity; the second case means $f=\int \tilde{f}$ for $\tilde{f} \in \mathscr{F}$, and (6) becomes the pure Baxter axiom (8) for $\tilde{f}$ and $g$.

Let us now look at some natural examples of integro-differential algebras, in addition to our standard examples $C^{\infty}(\mathbb{R})$ and $C^{\infty}[a, b]$.

Example 11. The analytic functions on the real interval $[a, b]$ form an integrodifferential subalgebra $C^{\omega}[a, b]$ of $C^{\infty}[a, b]$ over $K=\mathbb{R}$ or $K=\mathbb{C}$. It contains in turn the integro-differential algebra $K\left[x, e^{K x}\right]$ of exponential polynomials, defined as the space of all $K$-linear combinations of $x^{n} e^{\lambda x}$, with $n \in \mathbb{N}$ and $\lambda \in K$. Finally, the algebra of ordinary polynomials $K[x]$ is an integro-differential subalgebra in all cases. 
All the three examples above have algebraic analogs, with integro-differential structures defined in the expected way.

Example 12. For a field $K$ of characteristic zero, the formal power series $K[[x]]$ are an integro-differential algebra. One sets $\partial x^{k}=k x^{k-1}$ and $\int x^{k}=x^{k+1} /(k+1)$; note that the latter needs characteristic zero. The formal power series contain a highly interesting and important integro-differential subalgebra: the holonomic power series, defined as those whose derivatives span a finite-dimensional $K$-vector space $[29,77]$.

Of course $K[[x]]$ also contains (an isomorphic copy of) the integro-differential algebra of exponential polynomials. In fact, one can define $K\left[x, e^{K x}\right]$ algebraically as a quotient of the free algebra generated by the symbols $x^{k}$ and $e^{\lambda x}$, with $\lambda$ ranging over $K$. Derivation and integration are then defined in the obvious way. The exponential polynomials contain the polynomial ring $K[x]$ as an integro-differential subalgebra. When $K=\mathbb{R}$ or $K=\mathbb{C}$, we use the notation $K[x]$ and $K\left[x, e^{K x}\right]$ both for the analytic and the algebraic object since they are isomorphic.

The following example is a clever way of transferring the previous example to coefficient fields of positive characteristic.

Example 13. Let $K$ be an arbitrary field (having zero or positive characteristic). Then the algebra $H(K)$ of Hurwitz series [46] over $K$ is defined as the $K$-vector space of infinite $K$-sequences with the multiplication defined as

$$
\left(a_{n}\right) \cdot\left(b_{n}\right)=\left(\sum_{i=0}^{n}\left(\begin{array}{c}
n \\
i
\end{array}\right) a_{i} b_{n-i}\right)_{n}
$$

for all $\left(a_{n}\right),\left(b_{n}\right) \in H(K)$. If one introduces derivation and integration by

$$
\begin{aligned}
& \partial\left(a_{0}, a_{1}, a_{2}, \ldots\right)=\left(a_{1}, a_{2}, \ldots\right), \\
& \int\left(a_{0}, a_{1}, \ldots\right)=\left(0, a_{0}, a_{1}, \ldots\right),
\end{aligned}
$$

the Hurwitz series form an integro-differential algebra $\left(H(K), \partial, \int\right)$, as explained by [47] and [37]. Note that as an additive group, $H(K)$ coincides with the formal power series $K[[z]]$, but its multiplicative structure differs: We have an isomorphism

$$
\sum_{n=0}^{\infty} a_{n} z^{n} \mapsto\left(n ! a_{n}\right)
$$

from $K[[z]]$ to $H(K)$ if and only if $K$ has characteristic zero. The point is that one can integrate every element of $H(K)$, whereas the formal power series $z^{p-1}$ does not have an antiderivative in $K[[z]]$ if $K$ has characteristic $p>0$.

Now for the promised counterexample to the claim that the section axiom would suffice for merging a differential algebra $(\mathscr{F}, \partial)$ and a Rota-Baxter algebra $\left(\mathscr{F}, \int\right)$ into an integro-differential algebra $\left(\mathscr{F}, \partial, \int\right)$. 
Example 14. Set $R=K[y] /\left(y^{4}\right)$ for $K$ a field of characteristic zero and define $\partial$ on $\mathscr{F}=R[x]$ as usual. Then $(\mathscr{F}, \partial)$ is a differential algebra. Let us define a $K$-linear map $\int$ on $\mathscr{F}$ by

$$
\int f=\int^{*} f+f(0,0) y^{2},
$$

where $\int^{*}$ is the usual integral on $R[x]$ with $x^{k} \mapsto x^{k+1} /(k+1)$. Since the second term vanishes under $\partial$, we see immediately that $\int$ is a section of $\partial$. For verifying the pure Baxter axiom (8), we compute

$$
\begin{aligned}
& \left(\int f\right)\left(\int g\right)=\left(\int^{*} f\right)\left(\int^{*} g\right)+y^{2} \int^{*}(g(0,0) f+f(0,0) g)+f(0,0) g(0,0) y^{4}, \\
& \int f \int g=\int f\left(\int^{*} g+g(0,0) y^{2}\right)=\int^{*} f \int^{*} g+g(0,0) y^{2} \int^{*} f .
\end{aligned}
$$

Since $y^{4} \equiv 0$ and the ordinary integral $\int^{*}$ fulfills the pure Baxter axiom (8), this implies immediately that $\int$ does also. However, it does not fulfill the differential Baxter axiom (4) because it is not $\mathscr{C}$-linear: Observe that $\mathscr{C}$ is here $\operatorname{Ker}(\partial)=R$, so in particular we should have $\int(y \cdot 1)=y \cdot \int 1$. But one checks immediately that the left-hand side yields $x y$, while the right-hand side yields $x y+y^{3}$.

Ordinary Integro-Differential Algebras. The following example shows that our current notion of integro-differential algebra includes also algebras of "multivariate functions".

Example 15. Consider $\mathscr{F}=C^{\infty}\left(\mathbb{R}^{2}\right)$ with the derivation $\partial u=u_{x}+u_{y}$. Finding sections for $\partial$ means solving the partial differential equation $u_{x}+u_{y}=f$. Its general solution is given by

$$
u(x, y)=\int_{a}^{x} f(t, y-x+t) d t+g(y-x),
$$

where $g \in C^{\infty}(\mathbb{R})$ and $a \in \mathbb{R}$ are arbitrary. Let us choose $a=0$ for simplicity. In order to ensure a linear section, one has to choose $g=0$, arriving at

$$
\int f=\int_{0}^{x} f(t, y-x+t) d t,
$$

Using a change of variables, one may verify that $\int$ satisfies the pure Baxter axiom $(8)$, so $\left(\mathscr{F}, \int\right)$ is a Rota-Baxter algebra.

We see that the constant functions $\mathscr{C}=\operatorname{Ker}(\partial)$ are given by $(x, y) \mapsto c(x-y)$ with arbitrary $c \in C^{\infty}(\mathbb{R})$, while the initialized functions $\mathscr{I}=\operatorname{Im}\left(\int\right)$ are those $F \in \mathscr{F}$ that satisfy $F(0, y)=0$ for all $y \in \mathbb{R}$. In other words, $\mathscr{C}$ consists of all functions constant on the characteristic lines $x-y=$ const, and $\mathscr{I}$ of those satisfying the homogeneous initial condition on the vertical axis (which plays the role of a "noncharacteristic initial manifold"). This is to be expected since $\int$ integrates along the characteristic lines starting from the initial manifold. The evaluation $\mathbf{\varepsilon}: \mathscr{F} \rightarrow \mathscr{F}$ maps a function $f$ to the function $(x, y) \mapsto f(0, y-x)$. This means that $f$ is "sampled" only on the initial manifold, effectively becoming a univariate function: the general point $(x, y)$ is projected along the characteristics to the initial point $(0, y-x)$. 
Since $\mathbf{E}$ is multiplicative on $\mathscr{F}$, Lemma 3 tells us that $\left(\mathscr{F}, \partial, \int\right)$ is in fact an integro-differential algebra. Alternatively, note that $\mathscr{I}$ is an ideal and that $\int$ is $\mathscr{C}$ linear. Furthermore, we observe that here the polynomials are given by $K[x]$.

In the following, we want to restrict ourselves to boundary problems for ordinary differential equations. Hence we want to rule out cases like Example 15. The most natural way for distinguishing ordinary from partial differential operators is to look at their kernels since only the former have finite-dimensional ones. Note that in the following definition we deviate from the standard terminology in differential algebra [48, p. 58], where ordinary only refers to having a single derivation.

From now on, we restrict the ground ring $K$ to a field. We can now characterize when a differential algebra is ordinary by requiring that $\mathscr{C}$ be one-dimensional over $K$, meaning $\mathscr{C}=K$.

Definition 16. A differential algebra $(\mathscr{F}, \partial)$ is called ordinary if $\operatorname{dim}_{K} \operatorname{Ker}(\partial)=1$.

Note that except for Example 15 all our examples have been ordinary integrodifferential algebras. The requirement of ordinariness has a number of pleasant consequences. First of all, the somewhat tedious distinction between the weak and strong Baxter axioms disappears since now $\mathscr{F}$ is an algebra over its own field of constants $K=\mathscr{C}$. Hence $\int$ is by definition $\mathscr{C}$-linear, and Lemma 10 ensures that the pure Baxer axiom (8) is equivalent to the differential Baxter axiom (4). Let us summarize this.

Corollary 17. In an ordinary integro-differential algebra, the constant functions coincide with the ground field, and the strong and weak Baxter axioms are equivalent.

Recall that a character on an algebra (or group) is a multiplicative linear functional; this may be seen as a special case of the notion of character in representation theory, namely the case when the representation is one-dimensional. In our context, a character on an integro-differential algebra $\mathscr{F}$, is a $K$-linear map $\varphi: \mathscr{F} \rightarrow K$ satisfying $\varphi(f g)=\varphi(f) \varphi(g)$ and a fortiori also $\varphi(1)=1$. So we just require $\varphi$ to be a $K$-algebra homomorphism, as for example in [52, p. 407].

Ordinary integro-differential algebras will always have at least one character, namely the evaluation: One knows from Linear Algebra that a projector $P$ onto a one-dimensional subspace [w] of a $K$-vector space $V$ can be written as $P(v)=$ $\varphi(v) w$, where $\varphi: V \rightarrow K$ is the unique functional with $\varphi(w)=1$. If $V$ is moreover a $K$-algebra, a projector onto $K=[1]$ is canonically described by the functional $\varphi$ with normalization $\varphi(1)=1$. Hence multiplicative projectors like $\mathbf{E}$ can be viewed as characters. In the next section, we consider other characters on $\mathscr{F}$; for the moment let us note $\mathbf{E}$ as a distinguished character. We write $\mathscr{F} \bullet$ for the set of all nonzero characters on a $K$-algebra $\mathscr{F}$, in other words all algebra homomorphisms $\mathscr{F} \rightarrow K$.

One calls a $K$-algebra augmented if there exists a character on it. Its kernel $\mathscr{I}$ is then known as an augmentation ideal and forms a direct summand of $K$; see for example [32, p. 132]. Augmentation ideals are always maximal ideals (generalizing the $C^{\infty}[a, b]$ case) since the direct sum $\mathscr{F}=K+\mathscr{I}$ induces a ring isomorphism $\mathscr{F} / \mathscr{I} \cong K$. Corollary 4 immediately translates to the following characterization of integrals in ordinary differential algebras. 
Corollary 18. In an ordinary differential algebra $(\mathscr{F}, \partial)$, a section $\int$ of $\partial$ is an integral if and only if its evaluation is a character if and only if $\mathscr{I}=\operatorname{Im}\left(\int\right)$ is an augmentation ideal.

Initial Value Problems. It is clear that in general we cannot assume that the solutions of a differential equation with coefficients in $\mathscr{F}$ are again in $\mathscr{F}$. For example, in $\mathscr{F}=K[x]$, the differential equation $u^{\prime}-u=0$ has no solution. In fact, its "actual" solution space is spanned by $u(x)=e^{x}$ if $K=\mathbb{R}$ or $K=\mathbb{C}$. So in this case we should have taken the exponential polynomials $\mathscr{F}=K\left[x, e^{K x}\right]$ for ensuring that $u \in \mathscr{F}$. But if this is the case, we can also solve the inhomogeneous differential equation $u^{\prime}-u=f$ whose general solution is $K e^{x}+e^{x} \int e^{-x} f$, with $\int=\int_{0}^{x}$ as usual. Of course we can also incorporate the initial condition $u(0)=0$, which leads to $u=e^{x} \int e^{-x} f$.

This observation generalizes: Whenever we can solve the homogeneous differential equation within $\mathscr{F}$, we can also solve the initial value problem for the corresponding inhomogeneous problem. The classical tool for achieving this explicitly is the variation-of-constants formula [30, p. 74], whose abstract formulation is given in Theorem 20 below.

As usual [64], we will write $\mathscr{F}[\partial]$ for the ring of differential operators with coefficients in $\mathscr{F}$, see also Sect. 4. Let

$$
T=\partial^{n}+c_{n-1} \partial^{n-1}+\cdots+c_{0}
$$

be a monic (i.e. having leading coefficient 1 ) differential operator in $\mathscr{F}[\partial]$ of degree $n$. Then we call $u_{1}, \ldots, u_{n} \in \mathscr{F}$ a fundamental system for $T$ if it is a $K$-basis for $\operatorname{Ker}(T)$, so it yields the right number of solutions for the homogeneous differential equation $T u=0$. A fundamental system will be called regular if its associated Wronskian matrix

$$
W\left(u_{1}, \ldots, u_{n}\right)=\left(\begin{array}{ccc}
u_{1} & \cdots & u_{n} \\
u_{1}^{\prime} & \cdots & u_{n}^{\prime} \\
\vdots & \ddots & \vdots \\
u_{1}^{(n-1)} & \cdots & u_{n}^{(n-1)}
\end{array}\right)
$$

is invertible in $\mathscr{F}^{n \times n}$ or equivalently if its Wronskian $\operatorname{det} W\left(u_{1}, \ldots, u_{n}\right)$ is invertible in $\mathscr{F}$. Of course this alone implies already that $u_{1}, \ldots, u_{n}$ are linearly independent.

Definition 19. A monic differential operator $T \in \mathscr{F}[\partial]$ is called regular if it has a regular fundamental system.

For such differential operators, variation of constants goes through-the canonical initial value problem can be solved uniquely. This means in particular that regular differential operators are always surjective.

Theorem 20. Let $\left(\mathscr{F}, \partial, \int\right)$ be an ordinary integro-differential algebra. Given a regular differential operator $T \in \mathscr{F}[\partial]$ with $\operatorname{deg} T=n$ and a regular fundamental system $u_{1}, \ldots, u_{n} \in \mathscr{F}$, the canonical initial value problem 


$$
\begin{aligned}
& T u=f \\
& \mathbf{E} u=\mathbf{E} u^{\prime}=\cdots=\mathbf{E} u^{(n-1)}=0
\end{aligned}
$$

has the unique solution

$$
u=\sum_{i=1}^{n} u_{i} \int d^{-1} d_{i} f
$$

for every $f \in \mathscr{F}$, where $d=\operatorname{det} W\left(u_{1}, \ldots, u_{n}\right)$, and $d_{i}$ is the determinant of the matrix $W_{i}$ obtained from $W$ by replacing the $i$-th column by the $n$-th unit vector.

Proof. We can use the usual technique of reformulating $T u=f$ as a system of linear first-order differential equations with companion matrix $A \in \mathscr{F}^{n \times n}$. We extend the action of the operators $\int, \partial, \mathbf{E}$ componentwise to $\mathscr{F}^{n}$. Setting now

$$
\hat{u}=W \int W^{-1} \hat{f}
$$

with $\hat{f}=(0, \ldots, 0, f)^{\top} \in \mathscr{F}^{n}$, we check that $\hat{u} \in \mathscr{F}^{n}$ is a solution of the firstorder system $\hat{u}^{\prime}=A \hat{u}+\hat{f}$ with initial condition $\mathbf{E}(\hat{u})=0$. Indeed we have $\hat{u}^{\prime}=$ $W^{\prime} \int W^{-1} \hat{f}+W W^{-1} \hat{f}$ by the Leibniz rule and $A W=W^{\prime}$ since $u_{1}, \ldots, u_{n}$ are solutions of $T u=0$; so the differential system is verified. For checking the initial condition, note that $\mathbf{E} \int W^{-1} \hat{f}$ is already the zero vector, so we have also $\mathbf{E}(\hat{u})=0$ since $\mathbf{E}$ is multiplicative.

Writing $u$ for the first component of $\hat{u}$, we obtain a solution of the initial value problem (10), due to the construction of the companion matrix. Let us now compute $\hat{g}=W^{-1} \hat{f}$. Obviously $\hat{g}$ is the solution of the linear equation system $W \hat{g}=\hat{f}$. Hence Cramer's rule, which is also applicable for matrices over rings [53, p. 513], yields $\hat{g}_{i}$ as $d^{-1} d_{i} f$ and hence

$$
u=\left(W \int \hat{g}\right)_{1}=\sum_{i=1}^{n} u_{i} \int d^{-1} d_{i} f
$$

since the first row of $W$ is $\left(u_{1}, \ldots, u_{n}\right)$.

For proving uniqueness, it suffices to show that the homogeneous initial value problem only has the trivial solution. So assume $u$ solves (10) with $f=0$ and choose coefficients $c_{1}, \ldots, c_{n} \in K$ such that

$$
u=c_{1} u_{1}+\cdots+c_{n} u_{n} .
$$

Then the initial conditions yield $\mathbf{E}(W c)=0$ with $c=\left(c_{1}, \ldots, c_{n}\right)^{\top} \in K^{n}$. But we have also $\mathbf{E}(W c)=(\mathbf{E} W) c$ because $\mathbf{E}$ is linear, and $\operatorname{det} \mathbf{E} W=\mathbf{E}(\operatorname{det} W)$ because it is moreover multiplicative. Since $\operatorname{det} W \in \mathscr{F}$ is invertible, $\mathbf{E} W \in K^{n \times n}$ is regular, so $c=(\mathbf{E} W)^{-1} 0=0$ and $u=0$. 


\section{Integro-Differential Operators}

With integro-differential algebras, we have algebraized the functions to be used in differential equations and boundary problems, but we must also algebraize the operators inherent in both - the differential operators on the left-hand side of the former, and the integral operators constituting the solution of the latter. As the name suggests, the integro-differential operators provide a data structure that contains both of these operator species. In addition, it has as a third species the boundary operators needed for describing (global as well as local) boundary conditions of any given boundary problem for a LODE.

Definition. The basic idea is similar to the construction of the algebra of differential operators $\mathscr{F}[\partial]$ for a given differential algebra $(\mathscr{F}, \partial)$. But we are now starting from an ordinary integro-differential algebra $\left(\mathscr{F}, \partial, \int\right)$, and the resulting algebra of integro-differential operators will accordingly be denoted by $\mathscr{F}\left[\partial, \int\right]$. Recall that $\mathscr{F}[\partial]$ can be seen as the quotient of the free algebra generated by $\partial$ and $f \in \mathscr{F}$, modulo the ideal generated by the Leibniz rule $\partial f=f \partial+f^{\prime}$. For $\mathscr{F}\left[\partial, \int\right]$, we do the same but with more generators and more relations. In the following, all integrodifferential algebras are assumed to be ordinary.

Apart from $\int$, we will also allow a collection of "point evaluations" as new generators since they are needed for the specification of boundary problems. For example, the local boundary condition $u(1)=0$ on a function $u \in \mathscr{F}=C^{\infty}[0,1]$ gives rise to the functional $\mathbf{E}_{1} \in \mathscr{F}^{*}$ defined by $u \mapsto u(1)$. As one sees immediately, $\mathbf{E}_{1}$ is a character on $\mathscr{F}$, meaning $\mathbf{E}_{1}(u v)=\mathbf{E}_{1}(u) \mathbf{E}_{1}(v)$ for all $u, v \in \mathscr{F}$. This observation is the key for algebraizing "point evaluations" to an arbitrary integro-differential algebra where one cannot evaluate elements as in $C^{\infty}[0,1]$. We will see later how the characters serve as the basic building blocks for general local conditions like $3 u(\pi)-2 u(0)$ or global ones like $\int_{0}^{1} \xi u(\xi) d \xi$. Recall that we write $\mathscr{F} \bullet$ for the set of all characters on integro-differential algebra $\mathscr{F}$. In Sect. 3 we have seen that every integro-differential algebra $\left(\mathscr{F}, \partial, \int\right)$ contains at least one character, namely the evaluation $\mathbf{E}=1-\int \partial$ associated with the integral. Depending on the application, one may add other characters.

Definition 21. Let $\left(\mathscr{F}, \partial, \int\right)$ be an ordinary integro-differential algebra over a field $K$ and $\Phi \subseteq \mathscr{F}^{\bullet}$. The integro-differential operators $\mathscr{F}_{\Phi}\left[\partial, \int\right]$ are defined as the free $K$-algebra generated by $\partial$, and $\int$, the "functions" $f \in \mathscr{F}$, and the characters $\varphi \in \Phi \cup\{\mathbf{E}\}$, modulo the rewrite rules in Table 1. If $\Phi$ is understood, we write $\mathscr{F}\left[\partial, \int\right]$.

$$
\begin{array}{|l|l|l|}
\hline f g \rightarrow f \cdot g & \partial f \rightarrow f \partial+\partial \cdot f & \int f \int \rightarrow\left(\int \cdot f\right) \int-\int\left(\int \cdot f\right) \\
\varphi \psi \rightarrow \psi & \partial \varphi \rightarrow 0 & \int f \partial \rightarrow f-\int(\partial \cdot f)-(\mathbf{E} \cdot f) \mathbf{E} \\
\varphi f \rightarrow(\varphi \cdot f) \varphi & \partial \int \rightarrow 1 & \int f \varphi \rightarrow\left(\int \cdot f\right) \varphi \\
\hline
\end{array}
$$

Table 1 Rewrite Rules for Integro-Differential Operators 
The notation $U \cdot f$, used in the right-hand side of some of the rules above, refers to the action of $U \in \mathscr{F}\left\langle\partial, \int\right\rangle$ on a function $f \in \mathscr{F}$; in particular, $f \cdot g$ denotes the product of two functions $f, g \in \mathscr{F}$. It is an easy matter to check that the rewrite rules of Table 1 are fulfilled in $\left(\mathscr{F}, \partial, \int\right)$, so we may regard $\cdot$ as an action of $\mathscr{F}\left[\partial, \int\right]$ on $\mathscr{F}$. Thus every element $T \in \mathscr{F}\left[\partial, \int\right]$ acts as a map $T: \mathscr{F} \rightarrow \mathscr{F}$.

We have given the relations as a rewrite system, but their algebraic meaning is also clear: If in the free algebra $\mathscr{F}\left\langle\partial, \int\right\rangle$ of Definition 21 we form the two-sided ideal $\mathfrak{g}$ generated by the left-hand side minus right-hand side for each rule, then $\mathscr{F}_{\Phi}\left[\partial, \int\right]=\mathscr{F}\left\langle\partial, \int\right\rangle / \mathfrak{g}$. Note that there are infinitely many such rules since each choice of $f, g \in \mathscr{F}$ and $\varphi, \psi \in \Phi$ yields a different instance (there may be just finitely many characters in $\Phi$ but the coefficient algebra $\mathscr{F}$ is always infinite), so $\mathfrak{g}$ is an infinitely generated ideal (it was called the "Green's ideal" in [70] in a slightly more special setting). Note that one gets back the rewrite system of Table 1 if one uses the implied set of generators and a suitable ordering (see Sect. 7).

The reason for specifying $\mathfrak{g}$ via a rewrite system is of course that we may use it for generating a canonical simplifier for $\mathscr{F}\left[\partial, \int\right]$. This can be seen either from the term rewriting or from the Gröbner basis perspective: In the former case, we see Table 1 as a confluent and terminating rewrite system (modulo the ring axioms); in the latter case, as a noncommutative Gröbner basis with noetherian reduction (its elements are of course the left-hand side minus right-hand side for each rule). While we cannot give a detailed account of these issues here, we will briefly outline the Gröbner basis setting since our new proof in Sect. 7 will rely on it.

Noncommutative Gröbner Bases. As detailed in Section 2, it is necessary for our application to deal with infinitely generated ideals and an arbitrary set of indeterminates. The following description of such a noncommutative Gröbner basis setting is based on the somewhat dated but still highly readable Bergman paper [9]; for a summary see $[28, \S 3.3]$. For other approaches we refer the reader to $[61,62,85,86]$.

Let us first recall some notions for abstract reduction relations [4]. We consider a relation $\rightarrow \subseteq A \times A$ for a set $A$; typically $\rightarrow$ realizes a single step in a simplification process like the transformation of integro-differential operators according to Table 1 . The transitive closure of $\rightarrow$ is denoted by $\stackrel{+}{\rightarrow}$, its reflexive-transitive closure by $\stackrel{*}{\rightarrow}$. We call $a \in A$ irreducible if there is no $a_{0} \in A$ with $a \rightarrow a_{0}$; we write $A_{\downarrow}$ for the set of all irreducible elements. If $a \stackrel{*}{\rightarrow} a_{0}$ with $a_{0} \in A_{\downarrow}$, we call $a_{0}$ a normal form of $a$, denoted by $\downarrow a=a_{0}$ in case it is unique.

If all elements are to have a unique normal form, we have to impose two conditions: termination for banning infinite reductions and confluence reuniting forks. More precisely, $\rightarrow$ is called terminating if there are no infinite chains $a_{1} \rightarrow a_{2} \rightarrow \ldots$ and confluent if for all $a, a_{1}, a_{2} \in A$ the fork $a_{1} \stackrel{*}{\leftarrow} a \stackrel{*}{\rightarrow} a_{2}$ finds a reunion $a_{1} \stackrel{*}{\rightarrow} a_{0} \stackrel{*}{\leftarrow} a_{2}$ for some $a_{0} \in A$. If $\rightarrow$ is both terminating and confluent, it is called convergent.

Turning to noncommutative Gröbner bases theory, we focus on reduction relations on the free algebra $K\langle X\rangle$ over a commutative ring $K$ in an arbitrary set of indeterminates $X$; the corresponding monomials form the free monoid $\langle X\rangle$. Then a reduction system for $K\langle X\rangle$ is a set $\Sigma \subseteq\langle X\rangle \times K\langle X\rangle$ whose elements are called rules. For a rule $\sigma=(W, f)$ and monomials $A, B \in\langle X\rangle$, the $K$-module endomorphism of 
$K\langle X\rangle$ that fixes all elements of $\langle X\rangle$ except sending $A W B$ to $A f B$ is denoted by ${ }_{A} \sigma_{B}$ and called a reduction. It is said to act trivially on $a \in K\langle X\rangle$ if the coefficient of $A W B$ in $a$ is zero.

Every reduction system $\Sigma$ induces the relation $\rightarrow_{\Sigma} \subseteq K\langle X\rangle \times K\langle X\rangle$ defined by setting $a \rightarrow_{\Sigma} b$ if and only if $r(a)=b$ for some reduction acting nontrivially on $a$. We call its reflexive-transitive closure ${ }^{*}{ }_{\Sigma}$ the reduction relation induced by $\Sigma$, and we say that $a$ reduces to $b$ when $a{ }^{*}{ }_{\Sigma} b$. Accordingly we can speak of irreducible elements, normal forms, termination and confluence of $\Sigma$.

For ensuring termination, one can impose a noetherian monoid ordering on $\langle X\rangle$, meaning a partial ordering such that $1<A$ for all $A \in\langle X\rangle$ and such that $B<B^{\prime}$ implies $A B C<A B^{\prime} C$ for $A, B, B^{\prime}, C \in\langle X\rangle$. Recall that for partial (i.e. not necessarily total) orderings, noetherianity means that there are no infinite descending chains or equivalently that every nonempty set has a minimal element [8, p. 156]. Note that in a noetherian monoid ordering (like the divisibility relation on natural numbers), elements are not always comparable.

Now if one has a noetherian monoid ordering on $\langle X\rangle$, then $\Sigma$ will be terminating provided it respects $<$ in the sense that $W^{\prime}<W$ for every rule $(W, f) \in \Sigma$ and every nonzero monomial $W^{\prime}$ of $f$. (Let us also remark that the condition $1<A$ from above might as well be dropped, as in [9]: The given rewrite system cannot contain a rule $1 \rightarrow f$ since then $W<1$ for at least one nonzero monomial $W$ of $f$, so $1>W>W W>\cdots$ would yield an infinite descending chain. Such rules precluded, it is not stringent that constants in $K$ be comparable with the elements in $X$. But since it is nevertheless very natural and not at all restrictive, we stick to the monoid orderings as given above.)

It is typically more difficult to ensure confluence of a reduction system $\Sigma$. According to the definition, we would have to investigate all forks $a_{1} \stackrel{*}{\leftarrow} a \stackrel{*}{\rightarrow} a_{2}$, which are usually infinite in number. The key idea for a practically useful criterion is to consider only certain minimal forks (called ambiguities below, following Bergman's terminology) and see whether their difference eventually becomes zero. This was first described by Buchberger in [17] for the commutative case; see also [18, 21]. The general intuition behind minimal forks is analyzed in [20], where Gröbner bases are compared with Knuth-Bendix completion and Robinson's resolution principle.

An overlap ambiguity of $\Sigma$ is given by a quintuple $(\sigma, \tau, A, B, C)$ with $\Sigma$-rules $\sigma=(W, f), \tau=(V, g)$ and monomials $A, B, C \in\langle X\rangle \backslash\{1\}$ such that $W=A B$ and $V=B C$. Its associated S-polynomial is defined as $f C-A g$, and the ambiguity is called resolvable if the S-polynomial reduces to zero. (In general one may also have so-called inclusion ambiguities, but it turns out that one can always remove them without changing the resulting normal forms $[9, \S 5.1]$. Since the reduction system of Table 1 does not have inclusion ambiguities, we will not discuss them here.)

For making the connection to ideal theory, we observe that every reduction system $\Sigma$ gives rise to a two-sided ideal $I_{\Sigma}$ generated by all elements $W-f$ for $(W, f) \in \Sigma$; we have already seen this connection for the special case of the integrodifferential operators. Note that $a{ }_{\Sigma}^{*} 0$ is equivalent to $a \in I_{\Sigma}$.

In the given setting, the task of proving convergence can then be attacked by the so-called Diamond Lemma for Ring Theory, presented as Theorem 1.2 in Bergman's 
homonymous paper [9]; see also Theorem 3.21 in [28]. It is the noncommutative analog of Buchberger's criterion [18] for infinitely generated ideals. (In the version given below, we have omitted a fourth equivalent condition that is irrelevant for our present purposes.)

Theorem 22. Let $\Sigma$ be a reduction system for $K\langle X\rangle$ and $\leq$ a noetherian monoid ordering that respects $\Sigma$. Then the following conditions are equivalent:

- All ambiguities of $\Sigma$ are resolvable.

- The reduction relation ${ }^{*} \Sigma$ is convergent.

- We have the direct decomposition $K\langle X\rangle=K\langle X\rangle_{\downarrow}+I_{\Sigma}$ as $K$-modules.

When these conditions hold, the quotient algebra $K\langle X\rangle / I_{\Sigma}$ may be identified with the $K$-module $K\langle X\rangle_{\downarrow}$, having the multiplication $a \cdot b=\downarrow a b$.

We will apply Theorem 22 in Sect. 7 for proving that Table 1 constitutes a Gröbner basis for the ideal $\mathfrak{g}$. Hence we may conclude that $\mathscr{F}\left[\partial, \int\right]$ can be identified with the algebra $\mathscr{F}\left\langle\partial, \int\right\rangle_{\downarrow}$ of normal forms, and this is what gives us an algorithmic handle on the integro-differential operators. It is thus worth investigating these normal forms in some more detail.

Normal Forms. We start by describing a set of generators, which will subsequently be narrowed to the normal forms of $\mathscr{F}_{\Phi}\left[\partial, \int\right]$. The key observation is that in any monomial we never need more than one integration while all the derivatives can be collected at its end.

Lemma 23. Every integro-differential operator in $\mathscr{F}_{\Phi}\left[\partial, \int\right]$ can be reduced to a linear combination of monomials $f \varphi \int g \psi \partial^{i}$, where $i \geq 0$ and each of $f, \varphi, \int, g, \psi$ may also be absent.

Proof. Call a monomial consisting only of functions and functionals "algebraic". Using the left column of Table 1, it is immediately clear that all such monomials can be reduced to $f$ or $\varphi$ or $f \varphi$. Now let $w$ be an arbitrary monomial in the generators of $\mathscr{F}_{\Phi}\left[\partial, \int\right]$. By using the middle column of Table 1 , we may assume that all occurrences of $\partial$ are moved to the right, so that all monomials have the form $w=w_{1} \cdots w_{n} \partial^{i}$ with $i \geq 0$ and each of $w_{1}, \ldots, w_{n}$ either a function, a functional or $\int$. We may further assume that there is at most one occurrence of $\int$ among the $w_{1}, \ldots, w_{n}$. Otherwise the monomials $w_{1} \cdots w_{n}$ contain $\int \tilde{w} \int$, where each $\tilde{w}=f \varphi$ is an algebraic monomial. But then we can reduce

$$
\int \tilde{w} \int=\left(\int f \varphi\right) \int=\left(\int \cdot f\right) \varphi \int
$$

by using the corresponding rule of Table 1 . Applying these rules repeatedly, we arrive at algebraic monomials left and right of $\int$ (or just a single algebraic monomial if $\int$ is absent).

In TH $\exists \mathrm{OREM} \forall$, the integro-differential operators over an integro-differential algebra $\mathscr{F}$ of coefficient functions are built up by FreeIntDiffOp $[\mathscr{F}, \mathrm{K}]$. This functor constructs an instance of the monoid algebra with the word monoid over the 
infinite alphabet consisting of the letters $\partial$ and $\int$ along with a basis of $\mathscr{F}$ and with all multiplicative characters induced by evaluations at points in $\mathrm{K}$ :

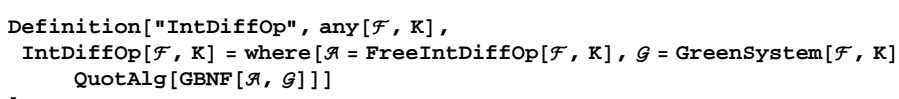

In this code fragment, the GreenSystem functor contains the encoding of the aforementioned rewrite system (Table 1), here understood as a noncommutative Gröbner basis. Normal forms for total reduction modulo infinite Gröbner bases are created by the GBNF functor, while the QuotAlg functor constructs the quotient algebra from the corresponding canonical simplifier (see Sect. 2 for details). For instance, multiplying the integral operator $\int$ by itself triggers an application of the Baxter rule:

$$
\begin{aligned}
& \text { Compute }\left[\left\langle\left\langle 1,\left\langle " \int "\right\rangle\right\rangle\right\rangle_{\text {II }}\left\langle\left\langle 1,\left\langle " \int "\right\rangle\right\rangle\right\rangle\right] / / \text { FormatP } \\
& -\mathrm{A} \mathrm{x}+\mathrm{xA}
\end{aligned}
$$

Here integral operators are denoted by A, following the notation in the older implementation [70].

We turn now to the normal forms of boundary conditions. Since they are intended to induce mappings $\mathscr{F} \rightarrow K$, it is natural to define them as those integro-differential operators that "end" in a character $\varphi \in \Phi$. For example, if $\varphi$ is the point evaluation $\mathbf{E}_{1}$ considered before, the composition $\mathbf{E}_{1} \partial$ describes the local condition $u^{\prime}(1)=$ 0 , the composition $\mathbf{E}_{1} \int$ the global condition $\int_{0}^{1} u(\xi) d \xi=0$. In general, boundary conditions may be arbitrary linear combinations of such composites; they are known as "Stieltjes conditions" in the literature $[15,16]$.

Definition 24. The elements of the right ideal

$$
\mid \Phi)=\Phi \cdot \mathscr{F}_{\Phi}\left[\partial, \int\right]
$$

are called boundary conditions over $\mathscr{F}$.

It turns out that their normal forms are exactly the linear combinations of local and global conditions as in the example mentioned above. As a typical representative over $\mathscr{F}=C^{\infty}(\mathbb{R})$, one may think of an element like

$$
\mathbf{E}_{0} \partial^{2}+3 \mathbf{E}_{\pi}-2 \mathbf{E}_{2 \pi} \int \sin x
$$

written as $u^{\prime \prime}(0)+3 u(\pi)-2 \int_{0}^{2 \pi} \sin \xi u(\xi) d \xi$ in traditional notation.

Proposition 25. Every boundary condition of $\mid \Phi)$ has the normal form

$$
\sum_{\varphi \in \Phi}\left(\sum_{i \in \mathbb{N}} a_{\varphi, i} \varphi \partial^{i}+\varphi \int f_{\varphi}\right)
$$

with only finitely many $a_{\varphi, i} \in K$ and $f_{\varphi} \in \mathscr{F}$ nonzero. 
Proof. By Lemma 23, every boundary condition of $\mid \Phi)$ is a linear combination of monomials having the form

$$
w=\chi f \varphi \int g \psi \partial^{i} \quad \text { or } \quad w=\chi f \varphi \partial^{i}
$$

where each of $f, g, \varphi, \psi$ may also be missing. Using the left column of Table 1 , the prefix $\chi f \varphi$ can be reduced to a scalar multiple of a functional, so we may as well assume that $f$ and $\varphi$ are not present; this finishes the right-hand case of (13). For the remaining case $w=\chi \int g \psi \partial^{i}$, assume first that $\psi$ is present. Then we have

$$
\chi\left(\int g \psi\right)=\chi\left(\int \cdot g\right) \psi=\left(\chi \int \cdot g\right) \chi \psi=\left(\chi \int \cdot g\right) \psi,
$$

so $w$ is again a scalar multiple of $\psi \partial^{i}$, and we are done. Finally, assume we have $w=\chi \int g \partial^{i}$. If $i=0$, this is already a normal form. Otherwise we obtain

$$
w=\chi\left(\int g \partial\right) \partial^{i-1}=(\chi \cdot g) \chi \partial^{i-1}-\chi \int g^{\prime} \partial^{i-1}-(\mathbf{E} \cdot g) \mathbf{E} \partial^{i-1},
$$

where the first and the last summand are in the required normal form, while the middle summand is to be reduced recursively, eventually leading to a middle term in normal form $\pm \chi \int g^{\prime} \partial^{0}= \pm \chi \int g^{\prime}$.

Most expositions of boundary problems - both analytic and numeric onesrestrict their attention to local conditions, even more specifically to those with just two point evaluation (so-called two-point boundary problems). While this is doubtless the most important case, there are at least three reasons for considering Stieltjes boundary conditions of the form (12).

- They arise in certain applications (e.g. heat radiated through a boundary) and in treating ill-posed problems by generalized Green's functions [70, p. 191].

- As we shall see (Sect. 5), they are needed for factoring boundary problems.

- Their algebraic description as a right ideal is very natural.

Hence we shall always mean all of $\mid \Phi)$ when we speak of boundary conditions.

Let us now turn to the other two ingredients of integro-differential operators: We have already mentioned the differential operators $\mathscr{F}[\partial]$, but we can now see them as a subalgebra of $\mathscr{F}_{\Phi}\left[\partial, \int\right]$. They have the usual normal forms since the Leibniz rule is part of the rewrite system. Analogously, we introduce the subalgebra of integral operators generated by the functions and $\int$. Using Lemma 23 , it is clear that the normal forms of integral operators are $\mathscr{F}$ itself and linear combinations of $f \int g$, and the only rule applicable to them is $\int f \int \rightarrow \cdots$ in Table 1 . Since we have already included $\mathscr{F}$ in $\mathscr{F}[\partial]$, we introduce $\mathscr{F}[S]$ as the $\mathscr{F}$-bimodule generated by $\int$ so that it contains only the monomials of the form $f \int g$.

Finally, we must consider the two-sided ideal $(\Phi)$ of $\mathscr{F}_{\Phi}\left[\partial, \int\right]$ generated by $\Phi$ whose elements are called boundary operators. A more economical description of $(\Phi)$ is as the left $\mathscr{F}$-submodule generated by $\mid \Phi)$ because by Lemma 23 any $w \chi \tilde{w}$ with $w, \tilde{w} \in \mathscr{F}\left[\partial, \int\right]$ can be reduced to $f \varphi \int g \psi \partial^{i} \chi \tilde{w}$. Note that $(\Phi)$ includes all finite dimensional projectors $P$ along Stieltjes boundary conditions. Any such projector 
can be described in the following way: If $u_{1}, \ldots, u_{n} \in \mathscr{F}$ and $\left.\beta_{1}, \ldots, \beta_{n} \in \mid \Phi\right)$ are biorthogonal (meaning $\beta_{i}\left(u_{j}\right)=\delta_{i j}$ ), then

$$
P=\sum_{i=1}^{n} u_{i} \beta_{i}: \mathscr{F} \rightarrow \mathscr{F}
$$

is the projector onto $\left[u_{1}, \ldots, u_{n}\right]$ whose kernel is the subspace of all $u \in \mathscr{F}$ such that $\beta(u)=0$ for all $\beta \in\left[\beta_{1}, \ldots, \beta_{n}\right]$. See for example [50, p. 71] or [66] for details. Note that all elements of $(\Phi)$ have the normal form (14), except that the $\left(u_{j}\right)$ need not be biorthogonal to the $\left(\beta_{i}\right)$.

We can now characterize the normal forms of $\mathscr{F}_{\Phi}\left[\partial, \int\right]$ in a very straightforward and intuitive manner: Every monomial is either a differential operator or an integral operator or a boundary operator. Hence every element of $\mathscr{F}_{\Phi}\left[\partial, \int\right]$ can be written uniquely as a sum $T+G+B$, with a differential operator $T \in \mathscr{F}[\partial]$, an integral operator $G \in \mathscr{F}\left[\int\right]$, and a boundary operator $B \in(\Phi)$.

Proposition 26. For an integro-differential algebra $\mathscr{F}$ and characters $\Phi \subseteq \mathscr{F} \bullet$ we have the direct decomposition $\mathscr{F}_{\Phi}\left[\partial, \int\right]=\mathscr{F}[\partial]+\mathscr{F}\left[\int\right]+(\Phi)$.

Proof. Inspection of Table 1 confirms that all integro-differential operators having the described sum representation $T+G+P$ are indeed in normal form. Let us now prove that every integro-differential operator of $\mathscr{F}_{\Phi}\left[\partial, \int\right]$ has such a representation. It is sufficient to consider its monomials $w$. If $w$ starts with a functional, we obtain a boundary condition by Proposition 25; so assume this is not the case. From Lemma 23 we know that

$$
w=f \varphi \int g \psi \partial^{i} \quad \text { or } \quad w=f \varphi \partial^{i},
$$

where each of $\varphi, g, \psi$ may be absent. But $w \in(\Phi)$ unless $\varphi$ is absent, so we may actually assume

$$
w=f \int g \psi \partial^{i} \quad \text { or } \quad w=f \partial^{i} .
$$

The right-hand case yields $w \in \mathscr{F}[\partial]$. If $\psi$ is present in the other case, we may reduce $\int g \psi$ to $\left(\int \cdot g\right) \psi$, and we obtain again $w \in(\Phi)$. Hence we are left with $w=$ $f \int g \partial^{i}$, and we may assume $i>0$ since otherwise we have $w \in \mathscr{F}\left[\int\right]$ immediately. But then we can reduce

$$
\begin{aligned}
w & =f\left(\int g \partial\right) \partial^{i-1}=f\left(g-\int(\partial \cdot g)-(\mathbf{E} \cdot g) \mathbf{E}\right) \partial^{i-1} \\
& =(f g) \partial^{i-1}-f \int(\partial \cdot g) \partial^{i-1}-(\mathbf{E} \cdot g) f \mathbf{E} \partial^{i-1}
\end{aligned}
$$

where the first term is obviously in $\mathscr{F}[\partial]$ and the last one in $(\Phi)$. The middle term may be reduced recursively until the exponent of $\partial$ has dropped to zero, leading to a term in $\mathscr{F}\left[\int\right]$.

We can observe the direct decomposition $\mathscr{F}_{\Phi}\left[\partial, \int\right]=\mathscr{F}[\partial] \dot{+} \mathscr{F}\left[\int\right] \dot{+}(\Phi)$ in the following sample multiplication of $\int \partial$ and $\partial \partial x e^{x} \int$ : 


$$
\begin{aligned}
& \text { Compute }\left[\left\langle\left\langle 1,\left\langle " \int ", " \partial "\right\rangle\right\rangle\right\rangle_{\text {II }}\left\langle\left\langle 1,\left\langle " \partial ", " \partial ",\left\langle "[1 ",\langle 1,1\rangle\rangle, " \int "\right\rangle\right\rangle\right\rangle\right] / /\right. \text { FormatP } \\
& -2 E+2 e^{x}+2 e^{x} x+2 e^{x} A+e^{x} x A+e^{x} \times D
\end{aligned}
$$

As in the previous computation, A stands for the integral $\int$, moreover $D$ for the derivation $\partial$, and $E$ for the evaluation. As we can see, the sum is composed of one differential operator (the last summand), two integral operators (in the middle), and three boundary operators (the first summands). Observe also that the input operators are not in normal form but the output operator is.

Basis Expansion. Regarding the canonical forms for $\mathscr{F}\left[\partial, \int\right]$, there is one more issue that we have so far swept under the rug. The problem is that in the current setup elements like $x \partial+3 x^{2} \partial$ and $\left(x+3 x^{2}\right) \partial$ are considered distinct normal forms. More generally, if $f+g=h$ holds in $\mathscr{F}$, there is no rule that allows us to recognize that $f+g \in \mathscr{F}\left[\partial, \int\right]$ and $h \in \mathscr{F}\left[\partial, \int\right]$ are the same. Analogously, if $\lambda \tilde{f}=\tilde{g}$ holds in $\mathscr{F}$ with $\lambda \in K$, then $\lambda \tilde{f}$ and $\tilde{g}$ are still considered to be different in $\mathscr{F}\left[\partial, \int\right]$. A slightly less trivial example is when $f=(\cos x)\left(\cos ^{2} x^{2}\right)$ and $g=-(\sin x)\left(\sin x^{2}\right)$ so that $h=\cos \left(x+x^{2}\right)$. What is needed in general is obviously a choice of basis for $\mathscr{F}$. But since such a choice is always to some degree arbitrary, we would like to postpone it as much as possible.

An unbiased way of introducing all $K$-linear relations in $\mathscr{F}$ is simply to collect them in all in the two-sided ideal

$$
\mathfrak{l}=(f+g-h, \lambda \tilde{f}-\tilde{g} \mid f+g=h \text { and } \lambda \tilde{f}=\tilde{g} \text { in } \mathscr{F}) \subseteq \mathscr{F}\left\langle\partial, \int\right\rangle,
$$

which we shall call the linear ideal. Since $\mathfrak{l}+\mathfrak{g}$ corresponds to a unique ideal $\mathfrak{l}$ in $\mathscr{F}\left[\partial, \int\right]$, the necessary refinement of $\mathscr{F}\left[\partial, \int\right]$ can now be defined as

$$
\mathscr{F}^{\#}\left[\partial, \int\right]=\mathscr{F}\left[\partial, \int\right] / \tilde{l} \cong \mathscr{F}\left\langle\partial, \int\right\rangle /(\mathfrak{l}+\mathfrak{g})
$$

whose elements shall be called expanded integro-differential operators. Note that $\tilde{\mathfrak{l}}$ is really the "same" ideal as $\mathfrak{l}$ except that now $f, g, h, \tilde{f}, \tilde{g} \in \mathscr{F}\left[\partial, \int\right]$. By the isomorphism above, coming from the Third Isomorphism Theorem [31, Thm 1.22], we can think of $\mathscr{F}^{\#}\left[\partial, \int\right]$ in two ways: Either we impose the linear relations on $\mathscr{F}\left[\partial, \int\right]$ or we merge them in with the Green's ideal-let us call these the a-posteriori and the combined approach, respectively.

For computational purposes, we need a ground simplifier on the free algebra [70, p. 525], which we define here as a $K$-linear canonical simplifier for $\mathscr{F}\left\langle\partial, \int\right\rangle / \mathfrak{l}$. Since all reduction rules of Table 1 are (bi)linear in $f, g \in \mathscr{F}$, any ground simplifier descends to a canonical simplifier $\sigma$ on $\mathscr{F}^{\#}\left[\partial, \int\right]$. In our implementations, $\sigma$ always operates by basis expansion (see below), but other strategies are conceivable. We can apply $\sigma$ either a-posteriori or combined:

- In the first case we apply $\sigma$ as a postprocessing step after computing the normal forms with respect to Table 1 . We have chosen this approach in the upcoming Maple implementation [49].

- In the combined approach, $\sigma$ may be used at any point during a reduction along the rules of Table 1 . It may be more efficient, however, to use $\sigma$ on the rules 
themselves to create a new reduction system on $\mathscr{F}\left\langle\partial, \int\right\rangle$; see below for an example. We have taken this approach in our earlier implementation $[69,70]$ and in the current implementation.

Generally the first approach seems to be superior, at least when $\sigma$ tends to create large expressions that are not needed for the rewriting steps of Table 1; this is what typically happens if the ground simplifier operates by basis expansion.

Assume now we choose a $K$-basis $\left(b_{i}\right)_{i \in I}$ of $\mathscr{F}$. If $\left(\hat{b}_{i}\right)_{i \in I}$ is the dual basis, we can describe the linear ideal more economically as

$$
\mathfrak{l}=\left(f-\sum_{i \in I} \hat{b}_{i}(f) b_{i} \mid f \in \mathscr{F}\right),
$$

so the linear basis $\left(b_{i}\right)_{i \in I}$ gives rise to an ideal basis for l. Its generators $f-\sum_{i} \cdots$ can be oriented to create a ground simplifier $\sigma: f \mapsto \sum_{i} \cdots$ effecting basis expansion.

If one applies now such a ground simplifier coming from a basis $\left(b_{i}\right)_{i \in I}$ in the combined approach, one can restrict the generators of $\mathscr{F}\left\langle\partial, \int\right\rangle$ to basis elements $b_{i} \in \mathscr{F}$ rather than all $f \in \mathscr{F}$, and the reduction rules can be adapted accordingly. For example, when $\mathscr{F}$ contains the exponential polynomials, the Leibniz rule $\partial f \rightarrow$ $f \partial+(\partial \cdot f)$ gets instantiated for $f=x e^{x}$ as $\partial\left(x e^{x}\right) \rightarrow\left(x e^{x}\right) \partial+e^{x}+x$, where the right-hand side now has three instead of two monomials! This is why the choice of basis was built into the definition of the precursor of $\mathscr{F} \#\left[\partial, \int\right]$ as in [70].

Before leaving this section on integro-differential operators, let us mention some interesting related work on these objects, carried out from a more algebraic viewpoint. In his papers [5, 6], Bavula establishes an impressive list of various (notably ring-theoretic) properties for algebras of integro-differential operators. The setup considered in these papers is, on the one hand, in many respects more general since it deals with partial rather than ordinary differential operators but, on the other hand, the coefficients are restricted to polynomials.

Seen from the more applied viewpoint taken here, the most significant difference is the lack of multiple point evaluations (and thus boundary conditions). Apart from these obvious differences, there is also a somewhat subtle difference in the meaning of $\mathbf{E}=1-\int \circ \partial$ that we have tried to elucidate in a skew-polynomial setting [67]. The upshot is that while our approach views $\mathbf{E}$ as a specific evaluation (the prototypical example is given after Definition 2), it does not have a canonical action in V. Bavula's setting (and neither in our skew-polynomial approach). This is a subtle but far-reaching difference that deserves future investigation.

\section{Applications for Boundary Problems}

In this section we combine the tools developed in the previous sections to build an algorithm for solving linear boundary problems over an ordinary integro-differential algebra; see also [73] for further details. We also outline a factorization method along a given factorization of the defining differential operator applicable to bound- 
ary problems for both linear ordinary and partial differential equations; see [66] in an abstract linear algebra setting and [74] for an overview.

For motivating our algebraic setting of boundary problems, let us consider our standard example of an integro-differential algebra $\left(\mathscr{F}, \partial, \int\right)$ with the integral operator

$$
\int: f \mapsto \int_{0}^{x} f(\xi) d \xi
$$

for $\mathscr{F}=C^{\infty}[0,1]$. The simplest two-point boundary problem reads then as follows: Given $f \in \mathscr{F}$, find $u \in \mathscr{F}$ such that

$$
\begin{aligned}
& u^{\prime \prime}=f \\
& u(0)=u(1)=0
\end{aligned}
$$

In this and the subsequent examples, we let $D$ and $A$ denote respectively the derivation $\partial$ and the integral operator $\int$. Moreover, we denote by $L$ the corresponding evaluation $\mathbf{E}$, which is the character

$$
L: f \mapsto f(0) .
$$

To express boundary problems we need additionally the evaluation at the endpoint of the interval

$$
R: f \mapsto f(1) .
$$

Note that $u$ is annihilated by any linear combination of these functionals so that problem (15) can be described by the pair $\left(D^{2},[L, R]\right)$, where $[L, R]$ is the subspace generated by $L, R$ in the dual space $\mathscr{F}^{*}$.

The solution algorithm presupposes a constructive fundamental system for the underlying homogeneous equation but imposes no other conditions (in the literature one often restricts to self-adjoint and/or second-order boundary problems). This is always possible (relative to root computations) in the important special case of LODEs with constant coefficient.

The Solution Algorithm. In the following, we introduce the notion of boundary problem in the context of ordinary integro-differential algebras. Unless specified otherwise, all integro-differential algebras in this section are assumed to be ordinary and over a fixed field $K$.

Definition 27. Let $\left(\mathscr{F}, \partial, \int\right)$ be an ordinary integro-differential algebra. Then a boundary problem of order $n$ is a pair $(T, \mathscr{B})$, where $T \in \mathscr{F}[\partial]$ is a regular differential operator of order $n$ and $\mathscr{B} \subseteq \mid \mathscr{F} \bullet)$ is an $n$-dimensional subspace of boundary conditions.

Thus a boundary problem is specified by a differential operator $T$ and a boundary space $\mathscr{B}=\left[\beta_{1}, \ldots, \beta_{n}\right]$ generated by linearly independent boundary conditions $\left.\beta_{1}, \ldots, \beta_{n} \in \mid \mathscr{F}^{\bullet}\right)$. In traditional notation, the boundary problem $(T, \mathscr{B})$ is then given by 


$$
\begin{aligned}
& T u=f, \\
& \beta_{1} u=\cdots=\beta_{n} u=0 .
\end{aligned}
$$

For a given boundary problem, we can restrict to a finite subset $\Phi \subseteq \mathscr{F} \bullet$, with the consequence that all subsequent calculations can be carried out in $\mathscr{F}_{\Phi}\left[\partial, \int\right]$ instead of $\mathscr{F}\left[\partial, \int\right]$. We will disregard this issue here for keeping the notation simpler.

Definition 28. A boundary problem $(T, \mathscr{B})$ is called regular if for each $f \in \mathscr{F}$ there exists a unique solution $u \in \mathscr{F}$ in the sense of (16).

The condition requiring $T$ to have the same order as the dimension of $\mathscr{B}$ in Definition 27 is only necessary but not sufficient for ensuring regularity: the boundary conditions might collapse on $\operatorname{Ker}(T)$. A simple example of such a singular boundary problem is $\left(-D^{2},[L D, R D]\right)$ using the notation from before; see also [70, p. 191] for more details on this particular boundary problem.

For an algorithmic test of regularity, one may also apply the usual regularity criterion for two-point boundary problems, as described in [66]. Taking any fundamental system of solutions $u_{1}, \ldots, u_{n}$ for the homogeneous equation, one can show that a boundary problem $(T, \mathscr{B})$ is regular if and only if the evaluation matrix

$$
\beta(u)=\left(\begin{array}{ccc}
\beta_{1}\left(u_{1}\right) & \cdots & \beta_{1}\left(u_{n}\right) \\
\vdots & \ddots & \vdots \\
\beta_{n}\left(u_{1}\right) & \cdots & \beta_{n}\left(u_{n}\right)
\end{array}\right) \in K^{n \times n}
$$

is regular.

For a regular boundary problem $(T, \mathscr{B})$, we can now define its Green's operator $G$ as the linear operator mapping a given forcing function $f \in \mathscr{F}$ to the unique solution $u \in \mathscr{F}$ of (16). It is characterized by the identities

$$
T G=1 \quad \text { and } \quad \operatorname{Im}(G)=\mathscr{B}^{\perp},
$$

where $\mathscr{B}^{\perp}=\{u \in \mathscr{F} \mid \beta(u)=0$ for all $\beta \in \mathscr{B}\}$ is the subspace of all "functions" satisfying the boundary conditions. We also write

$$
G=(T, \mathscr{B})^{-1}
$$

for the Green's operator of $(T, \mathscr{B})$.

The investigation of singular boundary problems (i.e. non-regular ones) is very enlightening but leads us too far afield; we shall investigate it at another junction. Let us just mention that it involves so-called modified Green's operators and functions $[80$, p. 216] and that is paves the way to an interesting non-commutative analog of the classical Mikusiński calculus [60].

We will now recast Theorem 20 in the language of Green's operators of initial value problems. Given a regular differential operator $T$ of order $n$, the theorem implies that the initial value problem $\left(T,\left[\mathbf{E}, \mathbf{E} \partial, \ldots, \mathbf{E} \partial^{n-1}\right]\right)$ is regular. We call its Green's operator the fundamental right inverse of $T$ and denote it by $T^{\star}$. 
Corollary 29. Let $\left(\mathscr{F}, \partial, \int\right)$ be an ordinary integro-differential algebra and let $T \in$ $\mathscr{F}[\partial]$ be a regular differential operator of order $n$ with regular fundamental system $u_{1}, \ldots, u_{n}$. Then its fundamental right inverse is given by

$$
T^{\Downarrow}=\sum_{i=1}^{n} u_{i} \int d^{-1} d_{i} \in \mathscr{F}\left[\partial, \int\right],
$$

where $d, d_{1}, \ldots, d_{n}$ are as in Theorem 20.

Before turning to the solution algorithm for boundary problems, let us also mention the following practical formula for specializing Corollary 29 to the important special case of LODEs with constant coefficients, which could also be proved directly e.g. via the Lagrange interpolation formula. For simplicity, we restrict ourselves to the case where the characteristic polynomial is separable.

Corollary 30. Let $\left(\mathscr{F}, \partial, \int\right)$ be an ordinary integro-differential algebra and consider the differential operator $T=\left(\partial-\lambda_{1}\right) \cdots\left(\partial-\lambda_{n}\right) \in \mathscr{F}[\partial]$ with $\lambda_{1}, \ldots, \lambda_{n} \in K$ mutually distinct. Assume each $u^{\prime}=\lambda_{i} u, \mathbf{E} \cdot u=1$ has a solution $u=e^{\lambda_{i} x} \in \mathscr{F}$ with reciprocal $u^{-1}=e^{-\lambda_{i} x} \in \mathscr{F}$. Then we have

$$
T^{\Downarrow}=\sum_{i=1}^{n} \mu_{i} e^{\lambda_{i} x} \int e^{-\lambda_{i} x}
$$

where $\mu_{i}^{-1}=\left(\lambda_{i}-\lambda_{1}\right) \cdots\left(\lambda_{i}-\lambda_{i-1}\right)\left(\lambda_{i}-\lambda_{i+1}\right) \cdots\left(\lambda_{i}-\lambda_{n}\right)$.

Proof. Let us write $V$ for the $n \times n$ Vandermonde determinant in $\lambda_{1}, \ldots, \lambda_{n}$ and $V_{i}$ for the $(n-1) \times(n-1)$ Vandermonde determinant in $\lambda_{1}, \ldots, \lambda_{i-1}, \lambda_{i+1}, \ldots, \lambda_{n}$. Evaluating the quantities of (17), one sees immediately that

$$
d=e^{\left(\lambda_{1}+\cdots \lambda_{n}\right) x} V \quad \text { and } \quad d_{i}=(-1)^{n+i} e^{\left(\lambda_{1}+\cdots+\lambda_{i-1}+\lambda_{i+1}+\cdots+\lambda_{n}\right) x} V_{i} .
$$

Hence we have $d_{i} / d=(-1)^{n+i} e^{-\lambda_{i} x} V_{i} / V$. Using the well-known formula for the Vandermonde determinant, one obtains $d_{i} / d=\mu_{i} e^{-\lambda_{i} x}$, and now the result follows from Corollary 29.

Summarizing our earlier results, we can now give a solution algorithm for computing $G=(T, \mathscr{B})^{-1}$, provided we have a regular fundamental system $u_{1}, \ldots, u_{n}$ for $T u=0$ and a $K$-basis $\beta_{1}, \ldots, \beta_{n}$ for $\mathscr{B}$. The algorithm proceeds in three steps:

1. Construct the fundamental right inverse $T^{\Downarrow} \in \mathscr{F}\left[\partial, \int\right]$ as in Corollary 29.

2. Determine the projector $P=\sum_{i=1}^{n} u_{i} \tilde{\beta}_{i} \in \mathscr{F}\left[\partial, \int\right]$ as in (14).

3. Compute $G=(1-P) T^{\Downarrow} \in \mathscr{F}\left[\partial, \int\right]$.

Theorem 31. The above algorithm computes the Green's operator $G \in \mathscr{F}\left[\partial, \int\right]$ for any regular boundary problem $(T, \mathscr{B})$.

Proof. See [73]. 
The computation of Green's operators for boundary problems for ODEs using the above algorithm takes on the following concrete form in $\mathrm{TH} \exists \mathrm{OREM} \forall$ code.

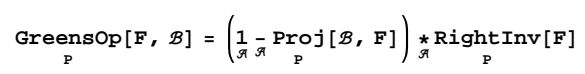

Here $\mathscr{B}$ is a basis for the boundary space and $\mathrm{F}$ a regular fundamental system.

Let us consider again example (15): Given $f \in \mathscr{F}$, find $u \in \mathscr{F}$ such that

$$
\begin{aligned}
& u^{\prime \prime}=f \\
& u(0)=u(1)=0
\end{aligned}
$$

The Green's operator $G$ of the boundary problem can be obtained by our implementation via the following computation

$$
\begin{aligned}
& \mathbf{G}=\text { Greensop }\left[D^{2},\langle L, R\rangle\right] \\
& -A x-x B+x A x+x B x
\end{aligned}
$$

where we use the notation from before: $A u=\int_{0}^{x} u(\xi) d \xi, L u=u(0), R u=u(1)$ and in addition, $B u=\int_{x}^{1} u(\xi) d \xi$. The corresponding Green's function is computed in an immediate postprocessing step:

$$
\begin{aligned}
& \text { GreensFct [G] } \\
& g[x, \xi]=\left\{\begin{array}{l}
-\xi+x \xi \Leftarrow \xi \leq x \\
-x+x \xi \Leftarrow x<\xi
\end{array}\right.
\end{aligned}
$$

As noted in [70], the Green's function provides a canonical form for the Green's operator. Moreover, one can obtain the function $u(x)$ and thus solve the boundary problem through knowledge of the Green's function in the following identity:

$$
u(x)=G f(x)=\int_{0}^{1} g(x, \xi) f(\xi) d \xi
$$

By replacing the Green's function obtained above in the latter integral we obtain

$$
u(x)=(x-1) \int_{0}^{x} \xi f(\xi) d \xi+x \int_{x}^{1}(\xi-1) f(\xi) d \xi .
$$

Furthermore, we can look at some specific instances of the forcing function $f(x)$. Let us first consider the simple example $f(x)=x$. By an immediate calculation, we obtain the expression for the action of $G$ on $f(x)$, which is $u(x)$ :

$$
\begin{aligned}
& \text { GreensOpAct }[G, \mathbf{x}] \\
& \frac{x}{6}+\frac{2 x^{4}}{3}+x^{3}\left(-\frac{5}{6}\right)
\end{aligned}
$$

The expression for the solution function $u(x)$ can easily become more complicated, as we can see in the next example, where we consider the instance

$$
f(x)=e^{2 x}+3 x^{2} \sin x^{3} .
$$


Relying on Mathematica for handling symbolic integration, we obtain:

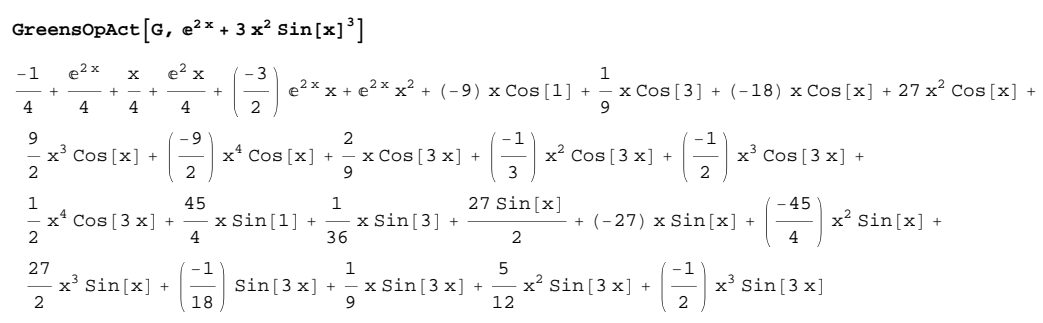

As a last example, let us consider $f(x)=\sin \sin x$. As we can notice below, it cannot be integrated with Mathematica:

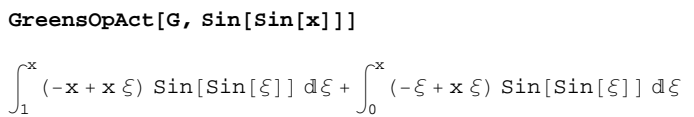

In order to carry out the integrals involved in the application of the Green's operator to a forcing function, one can use any numerical quadrature method (as also available in many computer algebra systems).

Composing and Factoring Boundary Problems. In the following, we discuss the composition of boundary problems corresponding to their Green's operators. We also describe how factorizations of a boundary problem along a given factorization of the defining operator can be characterized and constructed. We refer again to $[73,66]$ for further details. We assume that all operators are defined on suitable spaces such that the composition is well-defined. It is worth mentioning that the following approach works in an abstract setting, which includes in particular boundary problems for linear partial differential equations (LPDEs) and systems thereof; for simplicity, we will restrict ourselves in the examples to the LODE setting.

Definition 32. We define the composition of boundary problems $\left(T_{1}, \mathscr{B}_{1}\right)$ and $\left(T_{2}, \mathscr{B}_{2}\right)$ by

$$
\left(T_{1}, \mathscr{B}_{1}\right) \circ\left(T_{2}, \mathscr{B}_{2}\right)=\left(T_{1} T_{2}, \mathscr{B}_{1} \cdot T_{2}+\mathscr{B}_{2}\right) .
$$

So the boundary conditions from the first boundary problem are "translated" by the operator from the second problem. The composition of boundary problems is associative but in general not commutative. The next proposition tells us that the composition of boundary problems preserves regularity.

Proposition 33. Let $\left(T_{1}, \mathscr{B}_{1}\right)$ and $\left(T_{2}, \mathscr{B}_{2}\right)$ be regular boundary problems with Green's operators $G_{1}$ and $G_{2}$. Then $\left(T_{1}, \mathscr{B}_{1}\right) \circ\left(T_{2}, \mathscr{B}_{2}\right)$ is regular with Green's operator $G_{2} G_{1}$ so that

$$
\left(\left(T_{1}, \mathscr{B}_{1}\right) \circ\left(T_{2}, \mathscr{B}_{2}\right)\right)^{-1}=\left(T_{2}, \mathscr{B}_{2}\right)^{-1} \circ\left(T_{1}, \mathscr{B}_{1}\right)^{-1} .
$$

The simplest example of composing two boundary (more specifically, initial value) problems for ODEs is the following. Using the notation from before, one sees that 


$$
(D,[L]) \circ(D,[L])=\left(D^{2},[L D]+[L]\right)=\left(D^{2},[L, L D]\right) .
$$

Let now $(T, \mathscr{B})$ be a boundary problem and assume that we have a factorization $T=T_{1} T_{2}$ of the defining differential operator. We refer to [73, 66] for a characterization and construction of all factorizations

$$
(T, \mathscr{B})=\left(T_{1}, \mathscr{B}_{1}\right) \circ\left(T_{2}, \mathscr{B}_{2}\right)
$$

into boundary problems. In particular, if $(T, \mathscr{B})$ is regular, it can be factored into regular boundary problems: the left factor $\left(T_{1}, \mathscr{B}_{1}\right)$ is unique, while for the right factor $\left(T_{2}, \mathscr{B}_{2}\right)$ we can choose any subspace $\mathscr{B}_{2} \leq \mathscr{B}$ that makes the problem regular. We can compute the uniquely determined boundary conditions for the left factor by $\mathscr{B}_{1}=\mathscr{B} \cdot G_{2}$, where $G_{2}$ is the Green's operator for some regular right factor $\left(T_{2}, \mathscr{B}_{2}\right)$. By factorization, one can split a problem of higher order into subproblems of lower order, given a factorization of the defining operator. For algorithms and results about factoring ordinary differential operators we refer to [64, 78, 84].

Given a fundamental system of the differential operator $T$ and a right inverse of $T_{2}$, one can factor boundary problems in an algorithmic way as shown in [66] and in an integro-differential algebra [73]. As described in [74], we can also compute boundary conditions $\mathscr{B}_{2} \leq \mathscr{B}$ such that $\left(T_{2}, \mathscr{B}_{2}\right)$ is a regular right factor, given only a fundamental system of $T_{2}$. The unique left factor can be then computed as explained above. This allows us to factor a boundary problem if we can factor the differential operator and compute a fundamental system of only one factor. The remaining lower order problems can then also be solved by numerical methods.

Here is how we can compute the boundary conditions of the left and right factor problems for the boundary problem $\left(D^{2},[L, R]\right)$ from previous example (15), along the trivial factorization with $T_{1}=T_{2}=D$. The indefinite integral $A=\int_{0}^{x}$ is the Green's operator for the regular right factor $(D,[L])$.

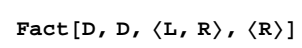

This factorization reads in traditional notation as

$$
\begin{array}{|l|l}
u^{\prime}=f \\
\int_{0}^{1} u(\xi) d \xi=0
\end{array} \circ \begin{aligned}
& u^{\prime}=f \\
& u(0)=0
\end{aligned}=\begin{aligned}
& u^{\prime \prime}=f \\
& u(0)=u(1)=0
\end{aligned} .
$$

Note that the boundary condition for the unique left factor is an integral (Stieltjes) boundary condition.

We consider as a second example the fourth order boundary problem [73, Ex. 33]:

$$
\begin{aligned}
& u^{\prime \prime \prime \prime}+4 u=f \\
& u(0)=u(1)=u^{\prime}(0)=u^{\prime}(1)=0 .
\end{aligned}
$$

Factoring the boundary problem along $D^{4}+4=\left(D^{2}-2 i\right)\left(D^{2}+2 i\right)$, we obtain the following boundary conditions for the factor problems. 


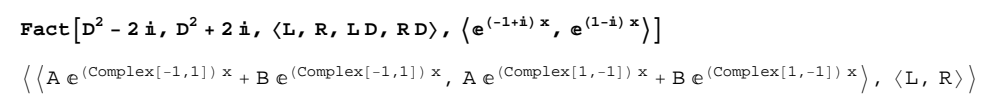

\section{Integro-Differential Polynomials}

In this section, we describe the algebra of integro-differential polynomials [72] obtained by adjoining an indeterminate function to a given integro-differential algebra $\left(\mathscr{F}, \partial, \int\right)$. Intuitively, these are all terms that one can build using an indeterminate $u$, coefficient functions $f \in \mathscr{F}$ and the operations $+, \cdot, \partial, \int$, identifying two terms if one can be derived from the other by the axioms of integro-differential algebras and the operations in $\mathscr{F}$. A typical term for $\left(K[x], \partial, \int\right)$ looks like this:

$$
\left(4 u u^{\prime} \int(x+3) u^{\prime 3}\right)\left(u^{\prime} \int u^{\prime \prime 2}\right)+\int x^{6} u u^{\prime \prime 5} \int\left(x^{2}+5 x\right) u^{3} u^{\prime 2} \int u
$$

From the computational point of view, a fundamental problem is to find a canonical simplifier (see Sect. 2) on these objects. For example, the above term can be transformed to

$$
\begin{aligned}
4 u u^{\prime 2} \int x u^{\prime 3} \int u^{\prime \prime 2}+4 u u^{\prime 2} \int u^{\prime 2} \int x u^{\prime 3} & +12 u u^{\prime 2} \int u^{\prime 3} \int u^{\prime \prime 2}+12 u u^{\prime 2} \int u^{\prime \prime 2} \int u^{\prime 3} \\
& +\int x^{6} u u^{\prime \prime 5} \int x^{2} u^{3} u^{\prime 2} \int u+5 \int x^{6} u u^{\prime \prime 5} \int x u^{3} u^{\prime 2} \int u
\end{aligned}
$$

by the Baxter axiom and the $K$-linearity of the integral.

As outlined in the next subsection, a notion of polynomial can be constructed for any variety in the sense of universal algebra. (In this general sense, an algebra is a set with an arbitrary number of operations, and a variety is a collection of such algebras satisfying a fixed set of identities. Typical examples are the varieties of groups, rings, and lattices.)

For sample computations in the algebra of integro-differential polynomials, we use a prototype implementation of integro-differential polynomials, based on the $\mathrm{TH} \exists \mathrm{OREM} \forall$ functor mechanism (see Sect. 2).

Polynomials in Universal Algebra. In this subsection, we describe the idea of the general construction of polynomials in universal algebra [45]. We refer to [54] for a comprehensive treatment; see also the surveys [1,27]. For the basic notions in universal algebra used below, see for example [4] or [33, Ch. 1].

Let $\mathscr{V}$ be a variety defined by a set $\mathscr{E}$ of identities over a signature $\Sigma$. Let $A$ be a fixed "coefficient domain" from the variety $\mathscr{V}$, and let $X$ be a set of indeterminates (also called "variables"). Then all terms in the signature $\Sigma$ with constants (henceforth referred to as coefficients) in $A$ and indeterminates in $X$ represent the same polynomial if their equality can be derived in finitely many steps from the identities in $\mathscr{E}$ and the operations in $A$. The set of all such terms $\mathscr{T}_{\Sigma}(A \cup X)$ modulo this congruence $\equiv$ is an algebra in $\mathscr{V}$, called the polynomial algebra for $\mathscr{V}$ in $X$ over $A$ and denoted by $A_{\mathscr{V}}[X]$. 
The polynomial algebra $A_{\mathscr{V}}[X]$ contains $A$ as a subalgebra, and $A \cup X$ is a generating set. As in the case of polynomials for commutative rings, we have the substitution homomorphism in general polynomial algebras. Let $B$ be an algebra in $\mathscr{V}$. Then given a homomorphism $\varphi_{1}: A \rightarrow B$ and a map $\varphi_{2}: X \rightarrow B$, there exists a unique homomorphism

$$
\varphi: A_{\mathscr{V}}[X] \rightarrow B
$$

such that $\varphi(a)=\varphi_{1}(a)$ for all $a \in A$ and $\varphi(x)=\varphi_{2}(x)$ for all $x \in X$. So in a categorical sense the polynomial algebra $A_{\mathscr{V}}[X]$ is a free product of the coefficient algebra $A$ and the free algebra over $X$ in $\mathscr{V}$; see also [1].

For computing with polynomials, we will construct a canonical simplifier on $A_{\mathscr{V}}[X]$ with associated system of canonical forms $\mathscr{R}$. As explained before (Sect. 2), the canonical simplifier provides for every polynomial in $A_{\mathscr{V}}[X]$, represented by some term $T$, a canonical form $R \in \mathscr{R}$ that represents the same polynomial, with different terms in $\mathscr{R}$ representing different polynomials; see also [54, p. 23].

The set $\mathscr{R}$ must be large enough to generate all of $A_{\mathscr{V}}[X]$ but small enough to ensure unique representatives. The latter requirement can be ensured by endowing a given set of terms with the structure of an algebra in the underlying variety.

Proposition 34. Let $\mathscr{V}$ be a variety over a signature $\Sigma$, let $A$ be an algebra in $\mathscr{V}$ and $X$ a set of indeterminates. If $\mathscr{R} \subseteq \mathscr{T}_{\Sigma}(A \cup X)$ is a set of terms with $A \cup X \subseteq \mathscr{R}$ that can be endowed with the structure of an algebra in $\mathscr{V}$, then different terms in $\mathscr{R}$ represent different polynomials in $A_{\mathscr{V}}[X]$.

Proof. Since $\mathscr{R}$ can be endowed with the structure of an algebra in the variety $\mathscr{V}$ and $A \cup X \subseteq \mathscr{R}$, there exists a unique substitution homomorphism

$$
\varphi: A_{\mathscr{V}}[X] \rightarrow \mathscr{R}
$$

such that $\varphi(a)=a$ for all $a \in A$ and $\varphi(x)=x$ for all $x \in X$. Let

$$
\pi: \mathscr{R} \rightarrow A_{\mathscr{V}}[X]
$$

denote the restriction of the canonical map associated with $\equiv$. Then we have $\varphi \circ$ $\pi(R)=R$ for all $R \in \mathscr{R}$, so $\pi$ is injective, and different terms in $\mathscr{R}$ indeed represent different polynomials.

As a well-known example, take the polynomial ring $R[x]$ in one indeterminate $x$ over a commutative ring $R$, which is $A_{\mathscr{V}}[X]$ for $A=R$ and $X=\{x\}$ with $\mathscr{V}$ being the variety of commutative unital rings. The set of all terms of the form $a_{n} x^{n}+\cdots+a_{0}$ with coefficients $a_{i} \in R$ and $a_{n} \neq 0$ together with 0 is a system of canonical forms for $R[x]$. One usually defines the polynomial ring directly in terms of these canonical forms. Polynomials for groups, bounded lattices and Boolean algebras are discussed in [54] along with systems of canonical forms.

Differential Polynomials. For illustrating the general construction described above, consider the algebra of differential polynomials over a commutative differential $K$ algebra $(\mathscr{F}, \partial)$ in one indeterminate function $u$, usually denoted by $\mathscr{F}\{u\}$. Clearly 
this is $A_{\mathscr{V}}[X]$ for $A=\mathscr{F}$ and $X=\{u\}$ with $\mathscr{V}$ being the variety of differential $K$ algebras. Terms are thus built up with the indeterminate $u$, coefficients from $\mathscr{F}$ and the operations $+, \cdot, \partial$; a typical example being

$$
\partial^{2}\left(f_{1} u^{2}+u\right) \partial\left(f_{2} u^{3}\right)+\partial^{3}\left(f_{3} u\right)
$$

By applying the Leibniz rule and the linearity of the derivation, it is clear that every polynomial is congruent to a $K$-linear combination of terms of the form

$$
f \prod_{i=0}^{\infty} u_{i}^{\beta_{i}}
$$

where $f \in \mathscr{F}$, the notation $u_{n}$ is short for $\partial^{n}(u)$, and only finitely many $\beta_{i} \in \mathbb{N}$ are nonzero. In the following, we use the multi-index notation $f u^{\beta}$ for terms of this form. For instance, $u^{(1,0,3,2)}$ is the multi-index notation for $u\left(u^{\prime \prime}\right)^{3}\left(u^{\prime \prime \prime}\right)^{2}$. The order of a differential monomial $u^{\beta}$ is given by the highest derivative appearing in $u^{\beta}$ or $-\infty$ if $\beta=0$.

Writing $\mathscr{R}$ for the set of all $K$-linear combinations of terms of the form (19), we already know that every polynomial is congruent to a term in $\mathscr{R}$. When $\mathscr{F}=K[x]$, a typical element of $\mathscr{R}$ is given by

$$
\left(3 x^{3}+5 x\right) u^{(1,0,3,2)}+7 x^{5} u^{(2,0,1)}+2 x u^{(1,1)} .
$$

To show that $\mathscr{R}$ is a system of canonical forms for $\mathscr{F}\{u\}$, by Proposition 34 it suffices to endow $\mathscr{R}$ with the structure of a commutative differential algebra. As a commutative algebra, $\mathscr{R}$ is just the polynomial algebra in the infinite set of indeterminates $u_{0}, u_{1}, u_{2}, \ldots$. For defining a derivation in a commutative algebra, by the Leibniz rule and $K$-linearity, it suffices to specify it on the generators. Thus $\mathscr{R}$ becomes a differential algebra by setting $\partial\left(u_{k}\right)=u_{k+1}$. One usually defines the differential polynomials directly in terms of these canonical forms, see for example [48].

Integro Polynomials. We outline the integro polynomials over a Rota-Baxter algebra as in Definition 8. This is related to the construction of free objects in general RotaBaxter algebras; we refer to [41] for details and references. By iterating the Baxter axiom (8), one obtains a generalization that is called the shuffle identity on $\mathscr{F}$ :

$$
\left(\int f_{1} \int \cdots \int f_{m}\right) \cdot\left(\int g_{1} \int \cdots \int g_{n}\right)=\sum \int h_{1} \int \cdots \int h_{m+n}
$$

Here the sum ranges over all shuffles of $\left(f_{1}, \ldots, f_{m}\right)$ and $\left(g_{1}, \ldots, g_{n}\right)$; see $[65,68,76]$ for details. The sum consists of $\left(\begin{array}{c}m+n \\ n\end{array}\right)$ shuffles, obtained by "shuffling" together $\int f_{1} \int \cdots \int f_{m}$ and $\int g_{1} \int \cdots \int g_{n}$ as words over the letters $\int f_{i}$ and $\int g_{j}$, such that the inner order in the words is preserved. For instance, we have

$$
\left(\int f_{1} \int f_{2}\right) \cdot\left(\int g_{1}\right)=\int f_{1} \int f_{2} \int g_{1}+\int f_{1} \int g_{1} \int f_{2}+\int g_{1} \int f_{1} \int f_{2} .
$$


for the simple $m=2, n=1$ case.

The integro polynomials over $\mathscr{F}$ are defined as $A_{\mathscr{V}}[X]$ for $A=\mathscr{F}$ and $X=\{u\}$ with $\mathscr{V}$ being the variety of Rota-Baxter algebras over $K$. The full construction of the canonical forms for integro polynomials is included in the following subsection. But it is clear that by expanding products of integrals by the shuffle identity, every integro polynomial is congruent to a $K$-linear combination of terms of the form

$$
f u^{k} \int f_{1} u^{k_{1}} \int \cdots \int f_{m} u^{k_{m}}
$$

with $f, f_{1}, \ldots, f_{m} \in \mathscr{F}$ and $k, k_{1}, \ldots, k_{m} \in \mathbb{N}$. However, they cannot be canonical forms, since terms like $\int(f+g) u$ and $\int f u+\int g u$ or $\int \lambda f u$ and $\lambda \int f u$ represent the same polynomials.

Writing $\mathscr{R}$ for the set of all $K$-linear combinations of terms of the form (21), the multiplication of two elements of $\mathscr{R}$ can now be defined via (20) as follows. Since the product of (21) with another term $g u^{l} \int g_{1} u^{l_{1}} \int \cdots \int g_{n} u^{l_{n}}$ should clearly be given by $f g u^{k+l}\left(\int f_{1} u^{k_{1}} \int \cdots \int f_{m} u^{k_{m}}\right)\left(\int g u^{l} g_{1} u^{l_{1}} \int \cdots f_{n} u^{l_{n}}\right)$, it remains to define the socalled shuffle product on integral terms (those having the form (21) with $f=1$ and $k=0$ ). This can be achieved immediately by using (20) with $f_{i} u^{k_{i}}$ and $g_{j} u^{l_{j}}$ in place of $f_{i}$ and $g_{j}$, respectively. It is easy to see that the shuffle product is commutative and distributive with respect to addition.

The shuffle product can also be defined recursively [68]. Let $J$ and $\tilde{J}$ range over integral terms (note that 1 is included as the special case of zero nested integrals). Then we have

$$
\left(\int f u^{k} J\right) \cdot\left(\int \tilde{f} u^{\tilde{k}} \tilde{J}\right)=\left(\int f u^{k}\right) \sqcup J \cdot\left(\int \tilde{f} u^{\tilde{k}} \tilde{J}\right)+\left(\int \tilde{f} u^{\tilde{k}}\right) \sqcup\left(\int f u^{k} J\right) \cdot \tilde{J},
$$

where $\sqcup: \mathscr{R} \times \mathscr{R} \rightarrow \mathscr{R}$ denotes the operation of nesting integrals (with the understanding that $\cdot$ binds stronger than $\sqcup$ ), defined on basis vectors by

$$
\int F_{1} \int \cdots \int F_{m} \sqcup \int G_{1} \int \cdots \int G_{n}=\int F_{1} \int \cdots \int F_{m} \int G_{1} \int \cdots \int G_{n},
$$

and extended bilinearly to all of $\mathscr{R}$. Here $F_{i}$ and $G_{j}$ stand for $f_{i} u^{k_{i}}$ and $g_{j} u^{l_{j}}$, respectively. For example, $\int F_{1} \int F_{2}$ and $\int G_{1}$ can be multiplied as

$$
\begin{array}{r}
\left(\int F_{1}\right) \sqcup\left(\int F_{2}\right) \cdot\left(\int G_{1}\right)+\left(\int G_{1}\right) \sqcup 1 \cdot\left(\int F_{1} \int F_{2}\right)=\left(\int F_{1}\right) \sqcup\left(\int F_{2} \int G_{1}+\int G_{1} \int F_{2}\right) \\
+\left(\int G_{1}\right) \sqcup\left(\int F_{1} \int F_{2}\right)=\int F_{1} \int F_{2} \int G_{1}+\int F_{1} \int G_{1} \int F_{2}+\int G_{1} \int F_{1} \int F_{2},
\end{array}
$$

analogous to the previous computation.

Representing Integro-Differential Polynomials. In the following, we describe in detail the universal algebra construction of the integro-differential polynomials and their canonical forms. We refer to $[39,40]$ for the related problem of constructing free objects in differential Rota-Baxter algebras. We consider the variety of integrodifferential algebras. Its signature $\Sigma$ contains: the ring operations, the derivation $\partial$, the integral $\int$, the family of unary "scalar multiplications" $(\cdot \lambda)_{\lambda \in K}$, and for convenience we also include the evaluation $\mathbf{\varepsilon}$. The identities $\mathscr{E}$ are those of a $K$-algebra, 
then $K$-linearity of the three operators $\partial, \int, \mathbf{E}$, the Leibniz rule (2), the section axiom (3), the Definition 2 of the evaluation, and the differential Baxter axiom (6).

Definition 35. Let $\left(\mathscr{F}, \partial, \int\right)$ be an integro-differential algebra. Then the algebra of integro-differential polynomials in $u$ over $\mathscr{F}$, denoted by $\mathscr{F}\{u\}$ in analogy to the differential polynomials, is the polynomial algebra $A_{\mathscr{V}}[X]$ for $A=\mathscr{F}$ and $X=\{u\}$ with $\mathscr{V}$ being the variety of integro-differential algebras over $K$.

Some identities following from $\mathscr{E}$ describe basic interactions between operations in $\mathscr{F}$ : the pure Baxter axiom (8), multiplicativity of the evaluation (5), the identities

$$
\mathbf{E}^{2}=\mathbf{E}, \quad \partial \mathbf{E}=0, \quad \mathbf{E} \int=0, \quad \int(\mathbf{E} f) g=(\mathbf{E} f) \int g,
$$

and the variant (7) of the differential Baxter axiom connecting all three operations.

We need to introduce some notational conventions. We use $f, g$ for coefficients in $\mathscr{F}$, and $V$ for terms in $\mathscr{T}_{\Sigma}(\mathscr{F} \cup\{u\})$. As for differential polynomials, we write $u_{n}$ for the $n$th derivative of $u$. Moreover, we write

$$
V(0) \text { for } \mathbf{E}(V) \text { and } u(0)^{\alpha} \text { for } \prod_{i=0}^{\infty} u_{i}(0)^{\alpha_{i}},
$$

where $\alpha$ is a multi-index.

As a first step towards canonical forms, we describe below a system of terms that is sufficient for representing every integro-differential polynomial (albeit not uniquely as we shall see presently).

Lemma 36. Every polynomial in $\mathscr{F}\{u\}$ can be represented by a finite K-linear combination of terms of the form

$$
f u(0)^{\alpha} u^{\beta} \int f_{1} u^{\gamma_{1}} \int \cdots \int f_{n} u^{\gamma_{n}}
$$

where $f, f_{1}, \ldots, f_{n} \in \mathscr{F}$, and each multi-index as well as $n$ may be zero.

Proof. The proof is done by induction on the structure of terms, using the above identities (8), (5), (20) and (24) of integro-differential algebras.

With the aid of the previous lemma we can determine the constants of $\mathscr{F}\{u\}$.

Proposition 37. Every constant in $\mathscr{F}\{u\}$ is represented as a finite sum $\sum_{\alpha} c_{\alpha} u(0)^{\alpha}$ with constants $c_{\alpha}$ in $\mathscr{F}$.

Proof. By the identity $\int \partial=1-\mathbf{\varepsilon}$, a term $V$ represents a constant in $\mathscr{F}\{u\}$ if and only if $\mathbf{E}(V) \equiv V$. Since $V$ is congruent to a finite sum of terms of the form (25) and since $\operatorname{Im}(\mathbf{E})=\mathscr{C}$, the identities for $\mathbf{E}$ imply that $V$ is congruent to a finite sum of terms of the form $c_{\alpha} u(0)^{\alpha}$.

The above representation (25) of the integro-differential polynomials is not unique since for example when trying to integrate differential polynomials by using integration by parts, terms like 


$$
\int f u^{\prime} \quad \text { and } \quad f u-\int f^{\prime} u-f(0) u(0)
$$

are equivalent. It becomes even more tedious to decide that, for instance,

$$
2 x u(0)^{(3,1)} u^{(1,3,0,4)} \int\left(2 x^{3}+3 x\right) u^{(1,2,3)} \int(x+2) u^{(2)}
$$

and

$$
\begin{gathered}
4 x u(0)^{(3,1)} u^{(1,3,0,4)} \int x^{3} u^{(1,2,3)} \int x u^{(2)}+6 x u(0)^{(3,1)} u^{(1,3,0,4)} \int x u^{(1,2,3)} \int(x+2) u^{(2)} \\
+12 x u(0)^{(3,1)} u^{(1,3,0,4)} \int x u^{(1,2,3)} \int u^{(2)}
\end{gathered}
$$

represent the same polynomial. In general, the following identity holds:

Lemma 38. We have

$$
\int V u_{k}^{\beta_{k}} u_{k+1} \equiv \frac{1}{\beta_{k}+1}\left(V u_{k}^{\beta_{k}+1}-\int V^{\prime} u_{k}^{\beta_{k}+1}-V(0) u_{k}(0)^{\beta_{k}+1}\right)
$$

where $k, \beta_{k} \geq 0$.

Proof. Using (7) and the Leibniz rule, the left-hand side becomes

$$
\int\left(V u_{k}^{\beta_{k}}\right)\left(u_{k}\right)^{\prime} \equiv V u_{k}^{\beta_{k}+1}-\int V^{\prime} u_{k}^{\beta_{k}+1}-\beta_{k} \int V u_{k}^{\beta_{k}} u_{k+1}-V(0) u_{k}(0)^{\beta_{k}+1},
$$

and the equation follows by collecting the $\int V u_{k}^{\beta_{k}} u_{k+1}$ terms.

The important point to note here is that if the highest derivative in the differential monomial $u^{\beta}$ of order $k+1$ appears linearly, then the term $\int f u^{\beta}$ is congruent to a sum of terms involving differential monomials of order at most $k$. This observation leads us to the following classification of monomials; confer also $[10,35]$.

Definition 39. A differential monomial $u^{\beta}$ is called quasiconstant if $\beta=0$, quasilinear if $\beta \neq 0$ and the highest derivative appears linearly; otherwise it is called functional. An integro-differential monomial (25) is classified according to its outer differential monomial $u^{\beta}$, and its order is defined to be that of $u^{\beta}$.

Proposition 40. Every polynomial in $\mathscr{F}\{u\}$ can be represented by a K-linear combination of terms of the form

$$
f u(0)^{\alpha} u^{\beta} \int f_{1} u^{\gamma_{1}} \int \cdots \int f_{n} u^{\gamma_{n}}
$$

where $f, f_{1}, \ldots, f_{n} \in \mathscr{F}$, the multi-indices $\alpha, \beta$ as well as $n$ may be zero and the $u^{\gamma_{1}}, \ldots, u^{\gamma_{n}}$ are functional.

Proof. By Lemma 36 we can represent every polynomial in $\mathscr{F}\{u\}$ as a $K$-linear combination of terms of the form

$$
f u(0)^{\alpha} u^{\beta} \int f_{1} u^{\gamma_{1}} \int \cdots \int f_{n} u^{\gamma_{n}}
$$


where the multi-indices and $n$ can also be zero. Let us first prove by induction on depth that every term can be written as in (28) but with nonzero multi-indices $\gamma_{k}$. The base case $n=1$ is trivial since $\int f_{1}$ can be pulled to the front. For the induction step we proceed from right to left, using the identity

$$
\int f \int V \equiv \int f \cdot \int V-\int V \int f
$$

implied by the pure Baxter axiom (8).

For proving that every multi-index $\gamma_{k}$ in (28) can be made functional, we use noetherian induction with respect to the preorder on $J=\int f_{1} u^{\gamma_{1}} \int \cdots \int f_{n} u^{\gamma_{n}}$ that first compares depth and then the order of $u^{\gamma_{1}}$. One readily checks that the left-hand side of (26) is greater than the right-hand side with respect to this preorder, provided that $V$ is of this form.

Applying Lemma 38 inductively, a term $\int f_{1} u^{\gamma_{1}}$ is transformed to a sum of terms involving only integral terms with functional differential monomials, and the base case $n=1$ follows. As induction hypothesis, we assume that all terms that are smaller than $J=f u(0)^{\alpha} u^{\beta} \int f_{1} u^{\gamma_{1}} \int \cdots \int f_{n} u^{\gamma_{n}}$ can be written as a sum of terms involving only functional monomials. Since $\int f_{2} u^{\gamma_{2}} \int \cdots \int f_{n} u^{\gamma_{n}}$ is smaller than $J$, it can be written as sum of terms involving only functional monomials; we may thus assume that $u^{\gamma_{2}}, \ldots, u^{\gamma_{n}}$ are all functional. Since $\gamma_{1}$ is nonzero, we are left with the case when $u^{\gamma_{1}}$ is quasilinear. Applying again Lemma 38 inductively, we can replace $u^{\gamma_{1}}$ in $J$ by a sum of terms involving only integral terms with functional differential monomials. The induction step follows then by the linearity of $\int$.

For implementing the integro-differential polynomials in TH $\exists \mathrm{OREM} \forall$ we use the functor hierarchy described in Sect. 2. The multi-index representation $u^{\beta}$ for terms of the form (19) is realized by the monoid $\mathbb{N}^{*}$ of natural tuples with finitely many nonzero entries, generated by a functor named TuplesMonoid. The nested integrals $\int f_{1} u^{\gamma_{1}} \int \cdots \int f^{n} u^{\gamma_{n}}$ are represented as lists of pairs of the form $\left\langle f_{k}, \gamma_{k}\right\rangle$, with $f_{k} \in \mathscr{F}$ and $\gamma_{k} \in \mathbb{N}^{*}$. The terms of the form (25) are then constructed via a cartesian product of monoids as follows:

Definition ["Term Monoid for IDP", any $[\mathcal{F}, \mathrm{N}]$,

TermMonoid $[\mathcal{F}, \mathbf{N}]=$ TuplesMonoid[N] $\times$ TuplesMonoid [N] $\times$ TuplesMonoid[F $\times$ TuplesMonoid $[N]]$

Using this construction, the integro-differential polynomials are built up by the functor FreeModule $[\mathscr{F}, \mathrm{B}]$ that constructs the $\mathscr{F}$-module with basis B. It is instantiated with $\mathscr{F}$ being a given integro-differential algebra and B the term monoid just described. We will equip this domain with the operations defined as below, using a functor named IntDiffPol $[\mathscr{F}, \mathrm{K}]$. Later in this section we will present some sample computations.

Canonical Forms for Integro-Differential Polynomials. It is clear that $K$-linear combinations of terms of the form (27) are still not canonical forms for the integrodifferential polynomials since by the linearity of the integral, terms like

$$
f \int(g+h) u \text { and } f \int g u+f \int h u
$$


or terms like

$$
f \int \lambda g u \text { and } \lambda f \int g u
$$

with $f, g, h \in \mathscr{F}$ and $\lambda \in K$ represent the same polynomial. To solve this problem, we can consider terms of the form (27) modulo these identities coming from linearity in the "coefficient" $f$ and the integral, in analogy to the ideal $\mathfrak{l}$ introduced in Sect. 4 for $\mathscr{F}^{\#}\left[\partial, \int\right]$. Confer also [39], where the tensor product is employed for constructing free objects in differential Rota-Baxter algebras. In the following, we assume for simplicity that $\mathscr{F}$ is an ordinary integro-differential algebra.

More precisely, let $\mathscr{R}$ denote the set of terms of the form (27) and consider the free $K$-vector space generated by $\mathscr{R}$. We identify terms

$$
f u(0)^{\alpha} u^{\beta} \int f_{1} u^{\gamma_{1}} \int \cdots \int f_{n} u^{\gamma_{n}}
$$

with the corresponding basis elements in this vector space. Then we factor out the subspace generated by the following identities (analogous to the construction of the tensor product):

$$
\begin{aligned}
& f U \int f_{1} u^{\gamma_{1}} \int \cdots \int\left(f_{k}+\tilde{f}_{k}\right) u^{\gamma_{k}} \int \cdots \int f_{n} u^{\gamma_{n}} \\
& \quad=f U \int f_{1} u^{\gamma_{1}} \int \cdots \int f_{k} u^{\gamma_{k}} \int \cdots \int f_{n} u^{\gamma_{n}}+f U \int f_{1} u^{\gamma_{1}} \int \cdots \int \tilde{f}_{k} u^{\gamma_{k}} \int \cdots \int f_{n} u^{\gamma_{n}} \\
& f U \int f_{1} u^{\gamma_{1}} \int \cdots \int\left(\lambda f_{k}\right) u^{\gamma_{k}} \int \cdots \int f_{n} u^{\gamma_{n}}=\lambda f U \int f_{1} u^{\gamma_{1}} \int \cdots \int f_{k} u^{\gamma_{k}} \int \cdots \int f_{n} u^{\gamma_{n}}
\end{aligned}
$$

Here $U$ is short for $u(0)^{\alpha} u^{\beta}$, and there are actually two more identities of the same type for ensuring $K$-linearity in $f$. We write $[\mathscr{R}]$ for this quotient space and denote the corresponding equivalence classes by

$$
\left[f u(0)^{\alpha} u^{\beta} \int f_{1} u^{\gamma_{1}} \int \cdots \int f_{n} u^{\gamma_{n}}\right] .
$$

By construction, the quotient module $[\mathscr{R}]$ now respects the linearity relations

$$
\begin{aligned}
& {\left[f U \int f_{1} u^{\gamma_{1}} \int \cdots \int\left(f_{k}+\tilde{f}_{k}\right) u^{\gamma_{k}} \int \cdots \int f_{n} u^{\gamma_{n}}\right]} \\
& \quad=\left[f U \int f_{1} u^{\gamma_{1}} \int \cdots \int f_{k} \int \cdots \int f_{n} u^{\gamma_{n}}\right]+\left[f U \int f_{1} u^{\gamma_{1}} \int \cdots \int \tilde{f}_{k} \int \cdots \int f_{n} u^{\gamma_{n}}\right] \\
& {\left[f U \int f_{1} u^{\gamma_{1}} \int \cdots \int\left(\lambda f_{k}\right) u^{\gamma_{k}} \int \cdots \int f_{n} u^{\gamma_{n}}\right]=\lambda\left[f U \int f_{1} u^{\gamma_{1}} \int \cdots \int f_{k} u^{\gamma_{k}} \int \cdots \int f_{n} u^{\gamma_{n}}\right] .}
\end{aligned}
$$

together with the ones for linearity in $f$.

As for the tensor product, we have canonical forms for the factor space by expanding the "coefficient" $f$ and all the $f_{k}$ in (29) with respect to a $K$-basis $\mathscr{B}$ for $\mathscr{F}$, assuming $\mathscr{B}$ contains 1 . Then every polynomial can be written as a $K$-linear combination of terms of the form

$$
b u(0)^{\alpha} u^{\beta} \int b_{1} u^{\gamma_{1}} \int \cdots \int b_{n} u^{\gamma_{n}}
$$

where $b, b_{1}, \ldots, b_{n} \in \mathscr{B}$ with the condition on multi-indices as in Proposition 40 .

To show that terms of the form (30) are canonical forms for the integro-differential polynomials, we endow the quotient space $[\mathscr{R}]$ with an integro-differential structure 
and invoke Proposition 34. For this we define the operations on the generators (29) and check that they respect the above linearity relations on $[\mathscr{R}]$.

First, we define a multiplication on $[\mathscr{R}]$. Let $\mathscr{R}_{0} \subseteq \mathscr{R}$ denote the $K$-subspace generated by integral terms $\int f_{1} u^{\gamma_{1}} \int \cdots \int f_{n} u^{\gamma_{n}}$, including $1 \in \mathscr{R}$ as the case $n=0$. Clearly, the nesting operation (23) can be defined in a completely analogous manner on such integral terms (the only difference being that we have now derivatives of the indeterminate). Since it is clearly $K$-linear, it induces an operation $\sqcup:\left[\mathscr{R}_{0}\right] \times$ $\left[\mathscr{R}_{0}\right] \rightarrow\left[\mathscr{R}_{0}\right]$. The next step is to define the shuffle product on $\mathscr{R}_{0}$ just as in (22), again with obvious modifications. Passing to the quotient yields the shuffle product $\cdot:\left[\mathscr{R}_{0}\right] \times\left[\mathscr{R}_{0}\right] \rightarrow\left[\mathscr{R}_{0}\right]$. This product is finally extending to a multiplication on all of $[\mathscr{R}]$ by setting

$$
\left[f u(0)^{\alpha} u^{\beta} J\right]\left[\tilde{f} u(0)^{\tilde{\alpha}} u^{\tilde{\beta}} \tilde{J}\right]=\left[f \tilde{f} u(0)^{\alpha+\tilde{\alpha}} u^{\beta+\tilde{\beta}}(J \cdot \tilde{J})\right]
$$

where $J$ and $\tilde{J}$ range over $\mathscr{R}_{0}$. Let us compute an example:

$$
\begin{aligned}
& \text { MultidD }\left[u[0]^{\langle 1\rangle} u^{\langle 2\rangle} " \int "(3 \mathbf{x}) u^{\langle 1,1\rangle} " \int " \mathbf{x}^{2} u^{\langle 0,2\rangle}, 3 u[0]^{\langle 2,3\rangle} u^{\langle 3,1\rangle} " \int " \mathbf{x} u^{\langle 1,0,1\rangle}\right] \\
& -18 u[0]^{\langle 3,3\rangle} u^{\langle 5,1\rangle} \int x u^{\langle 1,1\rangle} \int u^{\langle 0,2\rangle} \int x^{2} u^{\langle 0,2\rangle}-27 u[0]^{\langle 3,3\rangle} u^{\langle 5,1\rangle} \int x u^{\langle 1,1\rangle} \int x^{2} u^{\langle 0,2\rangle} \int u^{\langle 0,2\rangle}+ \\
& \quad 27 x u[0]^{\langle 3,3\rangle} u^{\langle 6,2\rangle} \int x u^{\langle 1,1\rangle} \int x^{2} u^{\langle 0,2\rangle}-\frac{9}{2} x u[0]^{\langle 3,3\rangle} u^{\langle 7,1\rangle} \int u^{\langle 0,2\rangle} \int x^{2} u^{\langle 0,2\rangle}- \\
& 27 u[0]^{\langle 4,4\rangle} u^{\langle 5,1\rangle} \int x u^{\langle 1,1\rangle} \int x^{2} u^{\langle 0,2\rangle}+\frac{3}{2} u[0]^{\langle 5,3\rangle} u^{\langle 5,1\rangle} \int u^{\langle 0,2\rangle} \int x^{2} u^{\langle 0,2\rangle}
\end{aligned}
$$

Since the multiplication on $\mathscr{F}$ and the shuffle product are commutative, associative, and distributive over addition, the multiplication on $[\mathscr{R}]$ is well-defined and gives $[\mathscr{R}]$ the structure of a commutative $K$-algebra.

The definition of a derivation $\partial$ on this algebra is straightforward, using the fact that it should respect $K$-linearity and the Leibniz rule (treating also the $u(0)^{\alpha}$ as constants), that it should restrict to the derivation on differential polynomials (which in turn restricts to the derivation on $\mathscr{F}$ ), and finally that it should also satisfy the section axiom (3). Here is a sample computation:

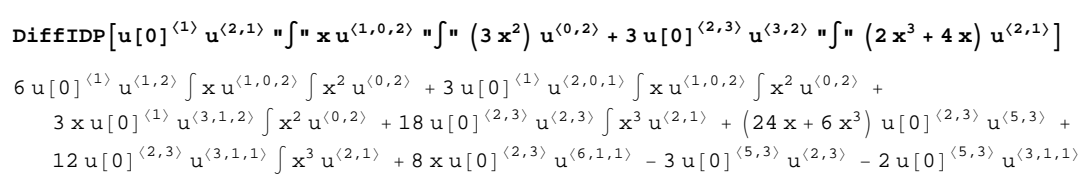

Using the $K$-linearity of this derivation, one verifies immediately that it is welldefined. From the definition it is clear that $K$-linear combinations of generators of the form $\left[u(0)^{\alpha}\right]$ are constants for $\partial$, and one can also check that all constants are actually of this form.

Finally, we define a $K$-linear integral on the differential $K$-algebra $([\mathscr{R}], \partial)$. Since we have to distinguish three different types of integrals, here and subsequently we will use the following notation: the usual big integral sign $\int$ for the integration to be defined, the small integral sign $\int$ for the elements of $\mathscr{R}$ as we have used it before, and $\int_{\mathscr{F}}$ for the integral on $\mathscr{F}$. 
The definition of the integral on $[\mathscr{R}]$ is recursive, first by depth and then by order of $u^{\beta}$, following the classification of monomials from Definition 39. In the base case of zero depth and order, we put

$$
\int\left[f u(0)^{\alpha}\right]=\left[\int_{\mathscr{F}} f\right]\left[u(0)^{\alpha}\right] .
$$

Turning to quasiconstant monomials, we use the following definition (which actually includes the base case when $J=1$ ):

$$
\int\left[f u(0)^{\alpha} J\right]=\left[u(0)^{\alpha}\left(\int_{\mathscr{F}} f\right) J\right]-\left[u(0)^{\alpha} \int\left(\int_{\mathscr{F}} f\right) J^{\prime}\right] .
$$

In the quasilinear case we write the generators in form

$$
\left[f u(0)^{\alpha} V u_{k}^{\beta_{k}} u_{k+1} J\right] \quad \text { with } \quad V=u_{0}^{\beta_{0}} \cdots u_{k-1}^{\beta_{k-1}}
$$

and construct the integral via (26). Writing $s=\beta_{k}+1$, we have $u_{k}^{\beta_{k}} u_{k+1}=\left(u_{k}^{s}\right)^{\prime} / s$, so we can define

$$
\int\left[f u(0)^{\alpha} V\left(u_{k}^{s}\right)^{\prime} J\right]=\left[f u(0)^{\alpha} V u_{k}^{s} J\right]-\left[u(0)^{\alpha}\right] \int[f V J]^{\prime}\left[u_{k}^{s}\right]-f(0)\left[u(0)^{\alpha+\beta} \hat{J}\right],
$$

where we write $f(0)$ for $\mathbf{E}(f)$ and $\hat{J}$ is 1 for $J=1$ and zero otherwise. In the functional case, we set

$$
\int\left[f u(0)^{\alpha} u^{\beta} J\right]=\left[u(0)^{\alpha} \int f u^{\beta} J\right]
$$

so here we can just let the integral sign slip into the equivalence class. One may check that the integral is well-defined in all the cases by an easy induction proof, using $K$-linearity of the integral, the evaluation on $\mathscr{F}$, and the derivation on $[\mathscr{R}]$.

Here is a small example of an integral computed in the quasiconstant case (note that IntIDP corresponds to the big integral and " $\int$ " to $\int$ in our notation):

$$
\begin{aligned}
& \text { IntIDP }\left[u[0]^{\langle 1\rangle} " \int " x u^{\langle 1,0,2\rangle} " \int "\left(x^{2}+2\right) u^{\langle 1,2\rangle}\right] \\
& 2 x u[0]^{\langle 1\rangle} \int x u^{\langle 1,0,2\rangle} \int u^{\langle 1,2\rangle}+x u[0]^{\langle 1\rangle} \int x u^{\langle 1,0,2\rangle} \int x^{2} u^{\langle 1,2\rangle}-2 u[0]^{\langle 1\rangle} \int x^{2} u^{\langle 1,0,2\rangle} \int u^{\langle 1,2\rangle}- \\
& u[0]^{\langle 1\rangle} \int x^{2} u^{\langle 1,0,2\rangle} \int x^{2} u^{\langle 1,2\rangle}
\end{aligned}
$$

The next example computes an integral in the quasilinear case:

$$
\begin{aligned}
& \text { IntIDP }\left[u[0]^{\langle 3,2\rangle} u^{\langle 2,1\rangle} " \int " \mathbf{x} \mathbf{u}^{\langle 1,0,2\rangle} " \int " \mathbf{x}^{2} \mathbf{u}^{\langle 1,1\rangle}\right] \\
& \frac{1}{6} u[0]^{\langle 5,2\rangle} \int x u^{\langle 4,0,2\rangle}-\frac{1}{6} x^{2} u[0]^{\langle 3,2\rangle} u^{\langle 2\rangle} \int x u^{\langle 4,0,2\rangle}+\frac{1}{6} x^{2} u[0]^{\langle 3,2\rangle} u^{\langle 5\rangle} \int x u^{\langle 1,0,2\rangle}- \\
& \frac{1}{6} u[0]^{\langle 5,2\rangle} u^{\langle 3\rangle} \int x u^{\langle 1,0,2\rangle}
\end{aligned}
$$

Note that all differential monomials within integrals are functional again, as it must be by our definition of $[\mathscr{R}]$.

By construction the integral defined above is a section of the derivation on $[\mathscr{R}]$. So for showing that $[\mathscr{R}]$ is an integro-differential algebra with operations, it remains 
only to prove the differential Baxter axiom (4). Equivalently, we can show that the evaluation

$$
\mathbf{E}=1-\int \partial
$$

is multiplicative by Corollary 4.

Recall that the algebra of constants $\mathscr{C}$ in $([\mathscr{R}], \partial)$ consists of $K$-linear combinations of generators of the form $\left[u(0)^{\alpha}\right]$. By a short induction proof, we see that

$$
\int\left[u(0)^{\alpha}\right][R]=\left[u(0)^{\alpha}\right] \int[R] .
$$

Hence the integral is homogeneous over the constants.

For showing that the evaluation is multiplicative, we first reassure ourselves that it operates in the expected way on integro-differential monomials.

Lemma 41. We have

$$
\mathbf{E}\left[f u(0)^{\alpha} u^{\beta} J\right]=f(0)\left[u(0)^{\alpha+\beta} \hat{J}\right],
$$

where $\hat{J}$ is 1 for $J=1$ and zero otherwise as in (33).

Proof. Note that $\mathbf{E}$ is $\mathscr{C}$-linear by (35), so we can omit the factor $u(0)^{\alpha}$. Assume first $\beta=0$. Then by the quasiconstant case (32) of the definition of the integral, we have

$$
\mathbf{E}[f J]=[f J]-\int[f J]^{\prime}=[f J]-\left[\left(\int_{\mathscr{F}} f^{\prime}\right) J\right]+\int\left[\left(\int_{\mathscr{F}} f^{\prime}\right) J^{\prime}\right]-\int\left[f J^{\prime}\right],
$$

which by $\int_{\mathscr{F}} f^{\prime}=f-f(0)$ gives

$$
f(0)[J]-f(0) \int[J]^{\prime}=f(0)[\hat{J}]
$$

because

$$
\int[J]^{\prime}=[J] \text { for } \quad J \neq 1
$$

by the functional case (34) and zero for $J=1$. If $\beta \neq 0$ is of order $k$, we write $u^{\beta}=V u_{k}^{s}$ with $s \neq 0$, and we compute

$$
\mathbf{E}\left[f u^{\beta} J\right]=\left[f V u_{k}^{s} J\right]-\int\left[f V u_{k}^{s} J\right]^{\prime}=f(0)\left[u(0)^{\beta} \hat{J}\right]
$$

by the quasilinear case (33) and the Leibniz rule.

Theorem 42. With the operations defined as above, $\left([\mathscr{R}], \partial, \int\right)$ has the structure of an integro-differential algebra.

Proof. As mentioned above, it suffices to prove that $\mathbf{E}$ is multiplicative, and we need only do this on the generators. Again omitting the $u(0)^{\alpha}$, we have to check that 


$$
\mathbf{E}\left[f u^{\beta} J\right]\left[\tilde{f} u^{\tilde{\beta}} \tilde{J}\right]=\mathbf{E}\left[f \tilde{f} u^{\beta+\tilde{\beta}}(J \cdot \tilde{J})\right]=\mathbf{E}\left[f u^{\beta} J\right] \cdot \mathbf{E}\left[\tilde{f} u^{\tilde{\beta}} \tilde{J}\right] .
$$

The case $J=\tilde{J}=1$ follows directly from Lemma 41 and the multiplicativity of $\mathbf{E}$ in $\mathscr{F}$. Otherwise the shuffle product $J \cdot \tilde{J}$ is a sum of integral terms, each of them unequal one. Using again Lemma 41 and the linearity of $\mathbf{E}$, the evaluation of this sum vanishes, as does $\mathbf{E}\left[f u^{\beta} J\right] \cdot \mathbf{E}\left[\tilde{f} u^{\tilde{\beta}} \tilde{J}\right]$.

Since $[\mathscr{R}]$ is an integro-differential algebra, we can conclude by Proposition 40 and Proposition 34 that $[\mathscr{R}]$ leads to canonical forms for integro-differential polynomials, up to the linearity relations: After a choice of basis, terms of the form (30) constitute a system of canonical forms for $\mathscr{F}\{u\}$. In the TH $\exists \mathrm{OREM} \forall$ implementation, we actually compute in $[\mathscr{R}]$ and do basis expansions only for deciding equality.

\section{From Rewriting to Parametrized Gröbner Bases}

Equipped with the integro-differential polynomials, we can now tackle the task of proving the convergence of the reduction rules in Table 1. As explained in Sect. 4, we will use the Diamond Lemma (Theorem 22) for this purpose. First of all we must therefore construct a noetherian monoid ordering $>$ on $\mathscr{F}\left\langle\partial, \int\right\rangle$ that is compatible with the reduction rules. In fact, there is a lot of freedom in defining such a $>$. It is sufficient to put $\partial>f$ for all $f \in \mathscr{F}$ and extend this to words by the graded lexicographic construction. The resulting partial ordering is clearly noetherian (since it is on the generators) and compatible with the monoid structure (by its grading). It is also compatible with the rewrite system because all rules reduce the word length except for the Leibniz rule, which is compatible because $\partial>f$.

Thus it remains to prove that all ambiguities of Table 1 are resolvable, and we have to compute the corresponding S-polynomials and reduce them to zero. On the face of it, there are of course infinitely many of these, suitably parametrized by $f, g \in \mathscr{F}$ and $\varphi, \psi \in \Phi$. For example, let us look at the minimal fork generated by $\int u \int v \int$. In this case, the rule $\int f \int$ may be applied either with $f=u$ or with $f=v$ yielding the reductions

$$
\begin{gathered}
\int u \int v \int \searrow \\
\left(\int \cdot u\right) \int v \int-\int\left(\int \cdot u\right) v \int \quad \int u\left(\int \cdot v\right) \int-\int u \int\left(\int \cdot v\right)
\end{gathered}
$$

with the S-polynomial $p=\left(\int \cdot u\right) \int v \int-\int\left(\int \cdot u\right) v \int-\int u\left(\int \cdot v\right) \int+\int u \int\left(\int \cdot v\right)$. But actually we should not call $p$ an S-polynomial since it represents infinitely many: one for each choice of $u, v \in \mathscr{F}$.

How should one handle this infinitude of S-polynomials? The problem is that for reducing S-polynomials like $p$ one needs not only the relations embodied in the reduction of Table 1 but also properties of the operations $\partial, \int: \mathscr{F} \rightarrow \mathscr{F}$ acting on $u, v \in \mathscr{F}$. Since these computations can soon become unwieldy, one should prefer a method that can be automated. There are two options that may be pursued: 
- Either one retreats to the viewpoint of rewriting, thinking of Table 1 as a twolevel rewrite system. On the upper level, it applies the nine parametrized rules with $f, g \in \mathscr{F}$ and $\varphi, \psi \in \Phi$ being arbitrary expressions. After each such step, however, there are additional reductions on the lower level for applying the properties of $\partial, \int: \mathscr{F} \rightarrow \mathscr{F}$ on these expressions. Using a custom-tailored reduction system for the lower level, this approach was used in the old implementation for generated an automated confluence proof [70].

- Or one views an S-polynomial like $p$ nevertheless as a single element, not in $\mathscr{F}\left\langle\partial, \int\right\rangle$ but in $\hat{F}\left\langle\partial, \int\right\rangle$ with $\hat{F}=\mathscr{F}\{u, v\}$. With this approach, one remains within the paradigm of parametrized Gröbner bases, and the interlocked operation of the two levels of reduction is clarified in a very coherent way: The upper level is driven by the canonical simplifier on $\hat{\mathscr{F}}\left[\partial, \int\right]$, the lower level by that on $\mathscr{F}\{u, v\}$.

It is the second approach that we will explain in what follows.

Using $\hat{\mathscr{F}}\left\langle\partial, \int\right\rangle$ instead of $\mathscr{F}\left\langle\partial, \int\right\rangle$ takes care of the parameters $f, g \in \mathscr{F}$ but then there are also the characters $\varphi, \psi \in \Phi$. The proper solution to this problem would be to use a refined version of integro-differential polynomials that starts from a whole family $\left(\int_{\varphi}\right)_{\varphi \in \Phi}$ of integrals instead of the single integral $\int$, thus leading to a corresponding family of evaluations $u(\varphi)$ instead of the single evaluation $u(0)$. We plan to pursue this approach in a forthcoming paper. For our present purposes, we can take either of the following positions:

- The characters $\varphi, \psi$ may range over an infinite set $\Phi$, but they are harmless since unlike the $f, g \in \mathscr{F}$ they do not come with any operations (whose properties must be accounted for by an additional level of reduction). In this case, Table 1 is still an infinitely generated ideal in $\hat{\mathscr{F}}\left\langle\partial, \int\right\rangle$, and we have to reduce infinitely many S-polynomials. But the ambiguities involving characters are all of a very simple nature, and their reduction of their S-polynomials is straightforward.

- Alternatively, we may restrict ourselves to a finite set of characters (as in most applications!) so that Table 1 actually describes a finitely generated ideal in $\hat{F}\left\langle\partial, \int\right\rangle$, and we need only consider finitely many S-polynomials.

The second alternative is somewhat inelegant due to the proliferation of instances for rules like $\varphi \psi \rightarrow \psi$. In our implementation, we have thus followed the first alternative with a straightforward treatment of parametrization in $\varphi, \psi$ but we will ignore this issue in what follows.

We can now use the new TH $\exists O R E M \forall$ implementation for checking that the nine rules in Table 1 form a Gröbner basis in $\hat{F}\left\langle\partial, \int\right\rangle$. As explained before, we use the Diamond Lemma for this purpose (note that the noetherian monoid ordering $>$ applies also to $\hat{\mathscr{F}}\left\langle\partial, \int\right\rangle$ except that we have now just two generators $u, v \in \hat{\mathscr{F}}=$ $\mathscr{F}\{u, v\}$ instead of all $f \in \mathscr{F}$ ). Hence it remains to check that all S-polynomials reduce to zero. We realize this by using the appropriate functor hierarchy, as follows. We first build up the algebra of the integro-differential polynomials having, in turn, integro-differential polynomials as coefficients, via the functor

$$
\text { IntDiffPolys[IntDiffPolys }[\mathscr{F}, \mathrm{K}], \mathrm{K}]
$$


and we denote the resulting domain by $\mathbb{P}$. Then we consider an instance of the functor constructing the integro-differential operators over $\mathbb{P}$. Finally, the computations are carried out over the algebra created by the GroebnerExtension functor taking the latter instance as input domain, that allows to perform polynomial reduction, S-polynomials and the Gröbner basis procedure.

Of course, the S-polynomials are generated automatically, but as a concrete example we check the minimal fork considered above:

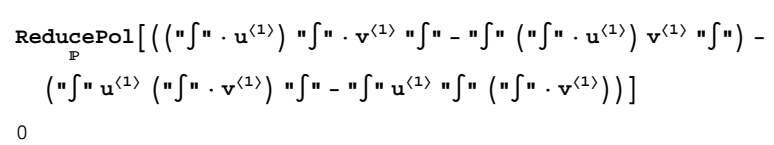

As it turns out, there are 17 nontrivial S-polynomials, and they all reduce to zero. This leads us finally to the desired convergence result for $\mathscr{F}\left[\partial, \int\right]$.

Theorem 43. The system of Table 1 represents a noncommutative Gröbner basis in $\mathscr{F}\left\langle\partial, \int\right\rangle$ for any graded lexicographic ordering satisfying $\partial>$ f for all $f \in \mathscr{F}$.

Proof. By the Diamond Lemma we must show that all S-polynomials $p \in \mathscr{F}\left\langle\partial, \int\right\rangle$ reduce to zero. Since they may contain at most two parameters $f, g \in \mathscr{F}$, let us write them as $p(f, g)$. But we have just observed that the corresponding S-polynomials $p(u, v) \in \hat{\mathscr{F}}\left\langle\partial, \int\right\rangle$ with $\hat{\mathscr{F}}=\mathscr{F}\{u, v\}$ reduce to zero. Using the substitution homomorphism

$$
\varphi: \hat{\mathscr{F}} \rightarrow \mathscr{F},(u, v) \mapsto(f, g),
$$

lifted to $\hat{\mathscr{F}}\left[\partial, \int\right] \rightarrow \mathscr{F}\left[\partial, \int\right]$ in the obvious way, we see that $p(f, g)=\varphi p(u, v)$ reduces to zero as well.

From the conclusion of the Diamond Lemma, we can now infer that Table 1 indeed establishes a canonical simplifier for $\mathscr{F}\left[\partial, \int\right]$.

\section{Conclusion}

The algebraic treatment of boundary problems is a new development in Symbolic Analysis that takes its starting point in differential algebra and enriches its structures by introducing an explicit notion of integration and boundary evaluations. Recall the three basic tools that we have introduced for this purpose:

- The category of integro-differential algebras $\left(\mathscr{F}, \partial, \int\right)$ for providing a suitable notion of "functions". (As explained in Sect. 2, here we do not think of categories and functors in the sense of Eilenberg and Maclane-this is also possible and highly interesting but must be deferred to another paper.)

- The functor creating the associated integro-differential operators $\mathscr{F}\left[\partial, \int\right]$ as a convenient language for expressing boundary problems (differential operators, boundary operators) and their solving Green's operators (integral operators). 
- The functor creating the associated integro-differential polynomials $\mathscr{F}\{u\}$, which describe the extension of an integro-differential algebra by a generic function $u$.

In each of these three cases, the differential algebra counterpart (i.e. without the "integro-") is well-known, and it appears as a rather simple substructure in the full structure. For example, the differential polynomials $\mathscr{F}\{u\}$ over a differential algebra $(\mathscr{F}, \partial)$ are simple to construct since the Leibniz rule effectively flattens out compound terms. This is in stark contrast to an integro-differential algebra $\left(\mathscr{F}, \partial, \int\right)$, where the Baxter rule forces the presence of nested integrals for ensuring closure under integration.

The interplay between these three basic tools is illustrated in a new confluence proof: For an arbitrary integro-differential algebra $\left(\mathscr{F}, \partial, \int\right)$, the rewrite system for the integro-differential operators $\mathscr{F}\left[\partial, \int\right]$ is shown to be a noncommutative Gröbner basis by the aid of the integro-differential polynomials $\mathscr{F}\{u, v\}$. Having a confluent rewrite system leads to a canonical simplifier, which is crucial for the algorithmic treatment as expounded in Sect. 2.

Regarding our overall mission - the algebraic treatment of boundary problems and integral operators-we have only scratched the surface, and much is left to be done. We have given a brief overview of solving, multiplying and factoring boundary problems in Sect. 5. But the real challenge lies ahead, namely how to extend our framework to:

- Linear Boundary Problems for LPDEs: As mentioned at the start of Sect. 5, the algebraic framework for multiplying and factoring boundary problems is set up to allow for LPDEs; see [66] for more details. But the problematic issue is how to design a suitable analog of $\mathscr{F}\left[\partial, \int\right]$ for describing integral and boundary operators (again the differential operators are obvious). This involves more than replacing $\partial$ by $\partial / \partial_{x}, \partial / \partial_{y}$ and $\int$ by $\int_{0}^{x}, \int_{0}^{y}$ because even the simplest Green's operators employ one additional feature: the transformation of variables, along with the corresponding interaction rules for differentiation (chain rule) and integration (substitution rule); see [74] for some first steps in this direction.

- Nonlinear Boundary Problems: A radically new approach is needed for that, so it seems appropriate to concentrate first on boundary problems for nonlinear ODEs and systems thereof. A natural starting point for such an investigation is the differential algebra setting, i.e. the theory of differential elimination $[44,11,12]$. By incorporating initial or boundary conditions, we can use explicit integral operators on equations, in addition to the usual differential operators (prolongations). As a consequence, the natural objects of study would no longer be differential but integro-differential polynomials.

We are well aware that such an approach will meet with many difficulties that will become manifest only as we progress. Nevertheless, we are confident that an algebraic — and indeed symbolic - treatment along these lines is possible.

Acknowledgements We acknowledge gratefully the support received from the SFB F013 in Subproject F1322 (principal investigators Bruno Buchberger and Heinz W. Engl), in earlier stages also 
Subproject F1302 (Buchberger) and Subproject F1308 (Engl). This support from the Austrian Science Fund (FWF) was not only effective in its financial dimension (clearly a necessary but not a sufficient condition for success), but also in a "moral" dimension: The stimulating atmosphere created by the unique blend of symbolic and numerical communities in this SFB-in particular the Hilbert Seminar mentioned in Sect. 1- has been a key factor in building up the raw material for our studies.

Over and above his general role in the genesis and evolution of the SFB F1322, we would like to thank Heinz W. Engl for encouragement, critical comments and helpful suggestions, not only but especially in the early stages of this project.

Loredana Tec is a recipient of a DOC-fFORTE-fellowship of the Austrian Academy of Sciences at the Research Institute for Symbolic Computation (RISC), Johannes Kepler University Linz. Georg Regensburger was partially supported by the Austrian Science Fund (FWF): J 3030-N18.

We would also like to thank an anonymous referee for giving us plenty of helpful suggestions and references that certainly increased the value of this article.

\section{References}

1. Aichinger, E., Pilz, G.F.: A survey on polynomials and polynomial and compatible functions. In: Proceedings of the Third International Algebra Conference, pp. 1-16. Kluwer Acad. Publ., Dordrecht (2003)

2. Albrecher, H., Constantinescu, C., Pirsic, G., Regensburger, G., Rosenkranz, M.: An algebraic operator approach to the analysis of Gerber-Shiu functions. Insurance Math. Econom. 46, 42$51(2010)$

3. Aschenbrenner, M., Hillar, C.J.: An algorithm for finding symmetric Gröbner bases in infinite dimensional rings. In: D. Jeffrey (ed.) Proceedings of ISSAC '08, pp. 117-123. ACM, New York, NY, USA (2008)

4. Baader, F., Nipkow, T.: Term rewriting and all that. Cambridge University Press, Cambridge (1998)

5. Bavula, V.V.: The algebra of integro-differential operators on a polynomial algebra (2009). URL http://arxiv.org/abs/0912.0723

6. Bavula, V.V.: The group of automorphisms of the algebra of polynomial integro-differential operators (2009). URL http: //arxiv.org/abs/0912.2537

7. Baxter, G.: An analytic problem whose solution follows from a simple algebraic identity. Pacific J. Math. 10, 731-742 (1960)

8. Becker, T., Weispfenning, V.: Gröbner bases, Graduate Texts in Mathematics, vol. 141. Springer, New York (1993). A computational approach to commutative algebra, In cooperation with Heinz Kredel

9. Bergman, G.M.: The diamond lemma for ring theory. Adv. in Math. 29(2), 178-218 (1978)

10. Bilge, A.H.: A REDUCE program for the integration of differential polynomials. Comput. Phys. Comm. 71(3), 263-268 (1992)

11. Boulier, F., Lazard, D., Ollivier, F., Petitot, M.: Representation for the radical of a finitely generated differential ideal. In: Proceedings of ISSAC '95, pp. 158-166. ACM, New York, NY, USA (1995)

12. Boulier, F., Ollivier, F., Lazard, D., Petitot, M.: Computing representations for radicals of finitely generated differential ideals. Appl. Algebra Engrg. Comm. Comput. 20(1), 73-121 (2009)

13. Bourbaki, N.: Algebra I. Chapters 1-3. Elements of Mathematics (Berlin). Springer-Verlag, Berlin (1998)

14. Brouwer, A.E., Draisma, J.: Equivariant Gröbner bases and the Gaussian two-factor model (2009). URL http://arxiv.org/abs/0908.1530

15. Brown, R.C., Krall, A.M.: Ordinary differential operators under Stieltjes boundary conditions. Trans. Amer. Math. Soc. 198, 73-92 (1974) 
16. Brown, R.C., Krall, A.M.: $n$-th order ordinary differential systems under Stieltjes boundary conditions. Czechoslovak Math. J. 27(1), 119-131 (1977)

17. Buchberger, B.: An algorithm for finding the bases elements of the residue class ring modulo a zero dimensional polynomial ideal (German). Ph.D. thesis, Univ. of Innsbruck (1965). English translation published in J. Symbolic Comput. 41(3-4), 475-511 (2006)

18. Buchberger, B.: Ein algorithmisches Kriterium für die Lösbarkeit eines algebraischen Gleichungssystems. Aequationes Math. 4, 374-383 (1970). English translation: An algorithmical criterion for the solvability of a system of algebraic equations. In: B. Buchberger, F. Winkler (eds.) Gröbner bases and applications, Cambridge Univ. Press (1998)

19. Buchberger, B.: A Critical-Pair/Completion Algorithm for Finitely Generated Ideals in Rings. In: E. Boerger, G. Hasenjaeger, D. Roedding (eds.) Logic and Machines: Decision Problems and Complexity, LNCS, vol. 171, pp. 137-161 (1984)

20. Buchberger, B.: History and basic features of the critical-pair/completion procedure. J. Symbolic Comput. 3(1-2), 3-38 (1987)

21. Buchberger, B.: Introduction to Gröbner bases. In: B. Buchberger, F. Winkler (eds.) Gröbner bases and applications. Cambridge Univ. Press (1998)

22. Buchberger, B.: Groebner Rings (2001). Contributed talk at International Conference on Computational Algebraic Geometry, University of Hyderabad, India

23. Buchberger, B.: Groebner rings and modules. In: S. Maruster, B. Buchberger, V. Negru, T. Jebelean (eds.) Proceedings of SYNASC 2001, pp. 22-25 (2001)

24. Buchberger, B.: Groebner Rings in Theorema: A Case Study in Functors and Categories. Tech. Rep. 2003-49, Johannes Kepler University Linz, Spezialforschungsbereich F013 (2003)

25. Buchberger, B.: Groebner bases in Theorema using functors. In: J. Faugere, D. Wang (eds.) Proceedings of SCC '08, pp. 1-15. LMIB Beihang University Press (2008)

26. Buchberger, B., Craciun, A., Jebelean, T., Kovacs, L., Kutsia, T., Nakagawa, K., Piroi, F., Popov, N., Robu, J., Rosenkranz, M., Windsteiger, W.: Theorema: Towards computer-aided mathematical theory exploration. J. Appl. Log. 4(4), 359-652 (2006)

27. Buchberger, B., Loos, R.: Algebraic simplification. In: Computer algebra, pp. 11-43. Springer, Vienna (1983)

28. Bueso, J., Gómez Torrecillas, J., Verschoren, A.: Algorithmic Methods in Non-Commutative Algebra: Applications to Quantum Groups. Springer (2003)

29. Chyzak, F., Salvy, B.: Non-commutative elimination in Ore algebras proves multivariate identities. J. Symbolic Comput. 26(2), 187-227 (1998)

30. Coddington, E.A., Levinson, N.: Theory of ordinary differential equations. McGraw-Hill Book Company, Inc., New York-Toronto-London (1955)

31. Cohn, P.M.: Introduction to Ring Theory. Springer (2000)

32. Cohn, P.M.: Basic algebra: Groups, Rings and Fields. Springer, London (2003)

33. Cohn, P.M.: Further algebra and applications. Springer-Verlag, London (2003)

34. Cucker, F., Shub, M. (eds.): Foundations of Computational Mathematics. Springer (1997). See http: / / www . focm. net/ for other FoCM based publications.

35. Gelfand, I.M., Diki1̆, L.A.: Fractional powers of operators, and Hamiltonian systems. Funkcional. Anal. i Priložen. 10(4), 13-29 (1976). English translation: Functional Anal. Appl. 10 (1976), no. 4, 259-273 (1977).

36. Grabmeier, J., Kaltofen, E., Weispfenning, V. (eds.): Computer algebra handbook. SpringerVerlag, Berlin (2003)

37. Guo, L.: Baxter algebras and differential algebras. In: Differential algebra and related topics (Newark, NJ, 2000), pp. 281-305. World Sci. Publ., River Edge, NJ (2002)

38. Guo, L.: What is... a Rota-Baxter algebra? Notices Amer. Math. Soc. 56(11), 1436-1437 (2009)

39. Guo, L., Keigher, W.: On differential Rota-Baxter algebras. J. Pure Appl. Algebra 212(3), 522-540 (2008)

40. Guo, L., Sit, W.Y.: Enumeration and generating functions of differential Rota-Baxter words. Math. Comput. Sci. (2011). URL http://dx.doi.org/10.1007/ s11786-010-0062-1 
41. Guo, L., Sit, W.Y.: Enumeration and generating functions of Rota-Baxter words. Math. Comput. Sci. (2011). URL http://dx.doi.org/10.1007/s11786-010-0061-2

42. Helton, J., Stankus, M.: NCGB 4.0: A noncommutative Gröbner basis package for mathematica (2010). URL http: / / www. math.ucsd. edu/ ncalg/

43. Hillar, C.J., Sullivant, S.: Finite Gröbner bases in infinite dimensional polynomial rings and applications (2009). URL http: / /arxiv.org/abs/0908.1777

44. Hubert, E.: Notes on triangular sets and triangulation-decomposition algorithms ii: Differential systems. In: U. Langer, F. Winkler (eds.) Symbolic and Numerical Scientific Computations, Lecture Notes in Computer Science, vol. 2630. Springer (2003)

45. Hule, H.: Polynome über universalen Algebren. Monatsh. Math. 73, 329-340 (1969)

46. Keigher, W.F.: On the ring of Hurwitz series. Comm. Algebra 25(6), 1845-1859 (1997)

47. Keigher, W.F., Pritchard, F.L.: Hurwitz series as formal functions. J. Pure Appl. Algebra 146(3), 291-304 (2000)

48. Kolchin, E.: Differential algebra and algebraic groups, Pure and Applied Mathematics, vol. 54. Academic Press, New York-London (1973)

49. Korporal, A., Regensburger, G., Rosenkranz, M.: A Maple package for integro-differential operators and boundary problems. ACM Commun. Comput. Algebra 44(3), 120-122 (2010). Also presented as a poster at ISSAC ' 10 .

50. Köthe, G.: Topological vector spaces (Volume I). Springer, New York (1969)

51. La Scala, R., Levandovskyy, V.: Letterplace ideals and non-commutative Gröbner bases. J. Symbolic Comput. 44(10), 1374-1393 (2009)

52. Lang, S.: Real and functional analysis, Graduate Texts in Mathematics, vol. 142. SpringerVerlag, New York (1993)

53. Lang, S.: Algebra, Graduate Texts in Mathematics, vol. 211, third edn. Springer-Verlag, New York (2002)

54. Lausch, H., Nöbauer, W.: Algebra of polynomials, North-Holland Mathematical Library, vol. 5. North-Holland Publishing Co., Amsterdam (1973)

55. Levandovskyy, V.: PLURAL, a non-commutative extension of SINGULAR: past, present and future. In: Mathematical software-ICMS 2006, LNCS, vol. 4151, pp. 144-157. Springer, Berlin (2006)

56. Levandovskyy, V.: Gröbner basis implementations: Functionality check and comparison. Website (2008). URL http://www.ricam.oeaw.ac.at/ Groebner-Bases-Implementations /

57. Madlener, K., Reinert, B.: Gröbner bases in non-commutative reduction rings. In: B. Buchberger, F. Winkler (eds.) Gröbner bases and applications, pp. 408-420. Cambridge Univ. Press, Cambridge (1998)

58. Madlener, K., Reinert, B.: String rewriting and Gröbner bases-a general approach to monoid and group rings. In: Symbolic rewriting techniques, Progr. Comput. Sci. Appl. Logic, vol. 15, pp. 127-180. Birkhäuser, Basel (1998)

59. Madlener, K., Reinert, B.: Non-commutative reduction rings. Rev. Colombiana Mat. 33(1), 27-49 (1999)

60. Mikusiński, J.: Operational calculus. Pergamon Press, New York (1959)

61. Mora, F.: Groebner bases for non-commutative polynomial rings. In: AAECC-3: Proceedings of the 3rd International Conference on Algebraic Algorithms and Error-Correcting Codes, pp. 353-362. Springer-Verlag, London, UK (1986)

62. Mora, T.: An introduction to commutative and noncommutative Gröbner bases. Theoret. Comput. Sci. 134(1), 131-173 (1994)

63. Nashed, M.Z., Votruba, G.F.: A unified operator theory of generalized inverses. In: M.Z. Nashed (ed.) Generalized inverses and applications (Proc. Sem., Math. Res. Center, Univ. Wisconsin, Madison, Wis., 1973), pp. 1-109. Academic Press, New York (1976)

64. van der Put, M., Singer, M.F.: Galois theory of linear differential equations, Grundlehren der Mathematischen Wissenschaften, vol. 328. Springer, Berlin (2003)

65. Ree, R.: Lie elements and an algebra associated with shuffles. Ann. of Math. (2) 68, 210-220 (1958) 
66. Regensburger, G., Rosenkranz, M.: An algebraic foundation for factoring linear boundary problems. Ann. Mat. Pura Appl. (4) 188(1), 123-151 (2009)

67. Regensburger, G., Rosenkranz, M., Middeke, J.: A skew polynomial approach to integrodifferential operators. In: J.P. May (ed.) Proceedings of ISSAC '09, pp. 287-294. ACM, New York, NY, USA (2009)

68. Reutenauer, C.: Free Lie algebras, vol. 7. The Clarendon Press Oxford University Press, New York (1993)

69. Rosenkranz, M.: The Green's algebra: A polynomial approach to boundary value problems. Phd thesis, Johannes Kepler University, Research Institute for Symbolic Computation (2003). Also available as RISC Technical Report 03-05, July 2003.

70. Rosenkranz, M.: A new symbolic method for solving linear two-point boundary value problems on the level of operators. J. Symbolic Comput. 39(2), 171-199 (2005)

71. Rosenkranz, M., Buchberger, B., Engl, H.W.: Solving linear boundary value problems via non-commutative Gröbner bases. Appl. Anal. 82, 655-675 (2003)

72. Rosenkranz, M., Regensburger, G.: Integro-differential polynomials and operators. In: D. Jeffrey (ed.) Proceedings of ISSAC '08, pp. 261-268. ACM, New York (2008)

73. Rosenkranz, M., Regensburger, G.: Solving and factoring boundary problems for linear ordinary differential equations in differential algebras. J. Symbolic Comput. 43(8), 515-544 (2008)

74. Rosenkranz, M., Regensburger, G., Tec, L., Buchberger, B.: A symbolic framework for operations on linear boundary problems. In: V.P. Gerdt, E.W. Mayr, E.H. Vorozhtsov (eds.) Computer Algebra in Scientific Computing. Proceedings of the 11th International Workshop (CASC 2009), LNCS, vol. 5743, pp. 269-283. Springer, Berlin (2009)

75. Rota, G.C.: Baxter algebras and combinatorial identities (I, II). Bull. Amer. Math. Soc. 75, 325-334 (1969)

76. Rota, G.C.: Ten mathematics problems I will never solve. Mitt. Dtsch. Math.-Ver. (2), 45-52 (1998)

77. Salvy, B., Zimmerman, P.: Gfun: a maple package for the manipulation of generating and holonomic functions in one variable. ACM Trans. Math. Softw. 20(2), 163-177 (1994)

78. Schwarz, F.: A factorization algorithm for linear ordinary differential equations. In: Proceedings of ISSAC ' 89 , pp. 17-25. ACM, New York (1989)

79. Seiler, W.: Computer algebra and differential equations: An overview. mathPAD 7, 34-49 (1997)

80. Stakgold, I.: Green's functions and boundary value problems. John Wiley \& Sons, New York (1979)

81. Stifter, S.: A generalization of reduction rings. J. Symbolic Comput. 4(3), 351-364 (1987)

82. Stifter, S.: Gröbner bases of modules over reduction rings. J. Algebra 159(1), 54-63 (1993)

83. Tec, L., Regensburger, G., Rosenkranz, M., Buchberger, B.: An automated confluence proof for an infinite rewrite system parametrized over an integro-differential algebra. In: K. Fukuda, J. van der Hoeven, M. Joswig, N. Takayama (eds.) Mathematical Software - Proceedings of ICMS 2010., LNCS, vol. 6327, pp. 245-248. Springer (2010)

84. Tsarev, S.P.: An algorithm for complete enumeration of all factorizations of a linear ordinary differential operator. In: Proceedings of ISSAC '96, pp. 226-231. ACM, New York (1996)

85. Ufnarovski, V.: Introduction to noncommutative Gröbner bases theory. In: B. Buchberger, F. Winkler (eds.) Gröbner bases and applications, pp. 259-280. Cambridge Univ. Press (1998)

86. Ufnarovskij, V.A.: Combinatorial and asymptotic methods in algebra. In: Algebra, VI, Encyclopaedia Math. Sci., vol. 57, pp. 1-196. Springer, Berlin (1995)

87. Windsteiger, W.: Building up hierarchical mathematical domains using functors in Theorema. Electr. Notes Theor. Comput. Sci. 23(3), 401-419 (1999) 